Homology, Homotopy and Applications, vol.16(1), 2014, pp.215-243

\title{
ON CONNECTIVE $K$-THEORY OF ELEMENTARY ABELIAN 2-GROUPS AND LOCAL DUALITY
}

\author{
GEOFFREY M.L. POWELL \\ (communicated by Nicholas J. Kuhn)
}

\begin{abstract}
The connective $k u$-(co)homology of elementary abelian 2groups is determined as a functor of the elementary abelian 2 -group, using the action of the Milnor operations $Q_{0}, Q_{1}$ on mod 2 group cohomology, the Atiyah-Segal theorem for $K U$ cohomology, together with an analysis of the functorial structure of the integral group ring; the functorial structure then reduces calculations to the rank 1 case.

These results are used to analyse the local cohomology spectral sequence calculating $k u$-homology, via a functorial version of local duality for Koszul complexes, giving a conceptual explanation of results of Bruner and Greenlees.
\end{abstract}

\section{Introduction}

The calculation of the $k u$-(co)homology of finite groups is interesting and highly non-trivial. The case of elementary abelian $p$-groups illustrates important features; these groups were first calculated by Ossa $[\mathbf{O s s 8 9}]$ and were studied further by Bruner and Greenlees [BG03], exhibiting a form of duality via local cohomology.

This paper shows how studying these as functors of the elementary abelian $p$ group $V$ gives a new and conceptual approach. The methods apply to any prime; 2 is privileged here since this requires an additional filtration argument when studying the local cohomology and also this is the case of interest when passing to $k o-(c o) h o m o l o g y$ (cf. [BG10] and [Pow12]). The first part of the paper gives complete descriptions of the functors $V \mapsto k u^{*}\left(B V_{+}\right)$and $V \mapsto k u_{*}\left(B V_{+}\right)$(see Theorems 5.13 and 5.16) as functors to graded $\mathbb{Z}[v]$-modules.

For $k u$-cohomology, the calculation relies on an analysis of the action of the Milnor operations $Q_{0}, Q_{1}$ on group cohomology $H \mathbb{F}_{2}^{*}\left(B V_{+}\right)$with $\mathbb{F}_{2}$ coefficients, together with the identification of the functor $V \mapsto K U^{0}\left(B V_{+}\right)$for periodic complex $K$-theory, provided by the Atiyah-Segal completion theorem. Whereas these are the ingredients to the classical approach using the Adams spectral sequence, a key step of the proof is simplified by using the functorial structure to reduce calculation to the rank 1 case,

This work was partly financed by the project ANR BLAN08-2 338236, HGRT.

Received October 22, 2012, revised January 6, 2014; published on May 27, 2014.

2010 Mathematics Subject Classification: 19L41, 20 J06.

Key words and phrases: connective $K$-theory, elementary abelian group, group cohomology, group homology, local cohomology.

Article available at http://dx.doi.org/10.4310/HHA.2014.v16.n1.a13

Copyright (C) 2014, International Press. Permission to copy for private use granted. 
$k u^{*}\left(B \mathbb{Z} / 2_{+}\right)$. This is a leitmotif throughout: functoriality sheds considerable light on the results.

To give a full functorial description, the integral group ring functor $V \mapsto \mathbb{Z}[V]$ is analysed, extending the results of Passi and others [PV77]. Of independent interest is the observation that the quotients which arise from studying the filtration of $\mathbb{Z}[V]$ by powers of the augmentation ideal are self-dual under Pontrjagin duality (see Theorems 3.13 and 3.11).

The second part of the paper applies these results to give an analysis of the local cohomology spectral sequence relating $k u^{*}\left(B V_{+}\right)$to $k u_{*}\left(B V_{+}\right)$; this gives insight into the description given by Bruner and Greenlees [BG03], notably on the origins of the differentials in the local cohomology spectral sequence. This is summarized in Theorem 8.23.

\section{Background}

\subsection{Definitions and notation}

Fix a prime $p$, and let $\mathbb{F}$ denote the prime field $\mathbb{F}_{p}, \mathscr{V}^{f}$ the full subcategory of finite-dimensional spaces in the category $\mathscr{V}$ of $\mathbb{F}$-vector spaces and $(-)^{\sharp}: \mathscr{V}^{\mathrm{op}} \rightarrow \mathscr{V}$ vector space duality.

Notation 2.1. 1. The category of functors from $\mathscr{V}^{f}$ to abelian groups is denoted $\mathscr{F} \mathscr{A}$, with $\mathscr{F}$ the full subcategory of functors with values in $\mathscr{V}$.

2. For $F$ in $\mathscr{F} \mathscr{A}, \bar{F}$ denotes the complementary constant-free summand so that $F$ splits canonically as $F(0) \oplus \bar{F}$, since $\mathscr{V}^{f}$ is pointed by 0 .

The categories $\mathscr{F} \mathscr{A}, \mathscr{F}$ are tensor abelian, with structure induced from $\mathscr{A} b$. (For basic properties of $\mathscr{F}$, see [Kuh94a, Kuh94b, Kuh95].) The Pontrjagin duality functor generalizes the duality for $\mathscr{F}$ introduced in [Kuh94a]:

Definition 2.2. Let $D: \mathscr{F} \mathscr{A}^{\mathrm{op}} \rightarrow \mathscr{F} \mathscr{A}$ be the functor

$$
D F(V):=\operatorname{Hom}_{\mathscr{A} b}\left(F\left(V^{\sharp}\right), \mathbb{Z} / p^{\infty}\right) \text {. }
$$

Recall that the socle of an object is its largest semi-simple sub-object and the head its largest semi-simple quotient.

Example 2.3. The symmetric powers, divided powers, and exterior powers are fundamental examples of (polynomial) functors in $\mathscr{F}$. For $n \in \mathbb{N}$, the $n$th symmetric power functor $S^{n}$ is defined by $S^{n}(V):=\left(V^{\otimes n}\right) / \mathfrak{S}_{n}$, the $n$th divided power functor by $\Gamma^{n}(V):=\left(V^{\otimes n}\right)^{\mathfrak{S}_{n}}$, and the $n$th exterior power functor identifies as $\Lambda^{n}(V) \cong$ $\left(V^{\otimes n} \otimes \operatorname{sign}\right)^{\mathfrak{S}_{n}}$. By convention, these functors are zero for negative integers $n$. There is a duality relation $S^{n} \cong D \Gamma^{n}$, whereas the functor $\Lambda^{n}$ is self-dual; for $p=2$, the functor $\Lambda^{n}$ is the head of $S^{n}$ and the socle of $\Gamma^{n}$.

These functors are examples of graded exponential functors; for example, the exponential structure induces natural coproducts $S^{n} \stackrel{\Delta}{\rightarrow} S^{i} \otimes S^{j}$ and products $S^{i} \otimes S^{j} \stackrel{\mu}{\rightarrow}$ $S^{n}$, for integers $i+j=n$.

Example 2.4. Yoneda's lemma provides the standard injective and projective objects of $\mathscr{F}$. The projective $P_{\mathbb{F}}$ is the functor $V \mapsto \mathbb{F}[V]$, which corepresents evaluation at 
$\mathbb{F}$; the injective $I_{\mathbb{F}}$ is given by $V \mapsto \mathbb{F}^{V^{\sharp}}$, representing $F \mapsto D F(\mathbb{F})$. Duality gives $I_{\mathbb{F}} \cong$ $D P_{\mathbb{F}}$, which relates the canonical decompositions $I_{\mathbb{F}} \cong \mathbb{F} \oplus \bar{I}_{\mathbb{F}}$ and $P_{\mathbb{F}} \cong \mathbb{F} \oplus \bar{P}_{\mathbb{F}}$, where $\mathbb{F}$ is the constant functor and $\bar{I}_{\mathbb{F}}$ (respectively $\bar{P}_{\mathbb{F}}$ ) is the complementary constant-free summand (see Notation 2.1).

The functor $I_{\mathbb{F}}$ is ungraded exponential and has associated diagonal $\Delta: I_{\mathbb{F}} \rightarrow I_{\mathbb{F}} \otimes$ $I_{\mathbb{F}}$ and multiplication $\mu: I_{\mathbb{F}} \otimes I_{\mathbb{F}} \rightarrow I_{\mathbb{F}}$; these are induced by applying the functor $I_{\mathbb{F}}$ to the sum $V \oplus V \rightarrow V$ and diagonal $V \rightarrow V \oplus V$, respectively, by using the natural isomorphism $\mathbb{F}^{U \oplus W} \cong \mathbb{F}^{U} \otimes \mathbb{F}^{W}$ for finite-dimensional spaces $U, W$.

By restriction and projection, these induce $\bar{I}_{\mathbb{F}} \stackrel{\bar{\Delta}}{\rightarrow} \bar{I}_{\mathbb{F}} \otimes \bar{I}_{\mathbb{F}}$ and $\bar{I}_{\mathbb{F}} \otimes \bar{I}_{\mathbb{F}} \stackrel{\bar{\mu}}{\rightarrow} \bar{I}_{\mathbb{F}}$, respectively; for $p=2$, these are the unique non-trivial morphisms of the given form. Dually, there is a product $\bar{\mu}: \bar{P}_{\mathbb{F}} \otimes \bar{P}_{\mathbb{F}} \rightarrow \bar{P}_{\mathbb{F}}$ and coproduct $\bar{\Delta}: \bar{P}_{\mathbb{F}} \rightarrow \bar{P}_{\mathbb{F}} \otimes \bar{P}_{\mathbb{F}}$.

Notation 2.5. For $0<n \in \mathbb{Z}$, let $\bar{P}_{\mathbb{F}}^{n}$ be the image of the iterated product $\bar{\mu}^{(n-1)}: \bar{P}_{\mathbb{F}}^{\otimes n}$ $\longrightarrow \bar{P}_{\mathbb{F}}$, and let $q_{n-1} \bar{P}_{\mathbb{F}}$ denote its cokernel, so that there is a short exact sequence

$$
0 \rightarrow \bar{P}_{\mathbb{F}}^{n} \rightarrow \bar{P}_{\mathbb{F}} \rightarrow q_{n-1} \bar{P}_{\mathbb{F}} \rightarrow 0 ;
$$

dually, let $p_{n-1} \bar{I}_{\mathbb{F}}$ denote the kernel of the iterated diagonal $\bar{I}_{\mathbb{F}} \stackrel{\bar{\Delta}^{(n-1)}}{\longrightarrow} \bar{I}_{\mathbb{F}}^{\otimes n}$.

Lemma 2.6 ([Kuh94b] $])$. Let $p=2$. For $n \in \mathbb{N}$ :

1. $\bar{I}_{\mathbb{F}}$ is the injective envelope of $\Lambda^{1}$, is uniserial, $\bar{I}_{\mathbb{F}} \cong \operatorname{colim}_{\rightarrow} p_{t} \bar{I}_{\mathbb{F}}$, and there are short exact sequences $0 \rightarrow p_{n} \bar{I}_{\mathbb{F}} \rightarrow p_{n+1} \bar{I}_{\mathbb{F}} \rightarrow \Lambda^{n+1} \rightarrow 0$;

2. $\bar{P}_{\mathbb{F}}$ is the projective cover of $\Lambda^{1}$, is uniserial, $\bar{P}_{\mathbb{F}} \cong \lim _{\leftarrow} q_{t} \bar{P}_{\mathbb{F}}$, and there are short exact sequences $0 \rightarrow \Lambda^{n+1} \rightarrow q_{n+1} \bar{P}_{\mathbb{F}} \rightarrow q_{n} \bar{P}_{\mathbb{F}} \rightarrow 0$;

3. $q_{n} \bar{P}_{\mathbb{F}}$ is the dual of $p_{n} \bar{I}_{\mathbb{F}}$.

The fact that $p_{n} \bar{I}_{\mathbb{F}}$ has a simple socle (for $n>0$ ) implies that it is easy to detect non-triviality of a sub-object by evaluating on $\mathbb{F}$, since $\Lambda^{i}(\mathbb{F})=\mathbb{F}$ for $i=1$ and is zero for $i>1$ :

Lemma 2.7. Let $p=2$. For $0<n \in \mathbb{N}$ :

1. if $G \subset p_{n} \bar{I}_{\mathbb{F}}$, then $G=0$ if and only if $G(\mathbb{F})=0$;

2. if $H \subset q_{n} \bar{P}_{\mathbb{F}}$, then $H=q_{n} \bar{P}_{\mathbb{F}}$ if and only if $H(\mathbb{F}) \neq 0$.

\section{The integral group ring functor}

Throughout this section, the prime $p$ is taken to be 2 .

3.1. The functors $\bar{P}_{\mathbb{Z}}, \bar{P}_{\mathbb{Z}_{2}}$

Notation 3.1. 1. Let $P_{\mathbb{Z}}$ denote the integral group ring functor $V \mapsto \mathbb{Z}[V]$, and let $\bar{P}_{\mathbb{Z}}$ denote the augmentation ideal, so that there is a direct sum decomposition $P_{\mathbb{Z}} \cong \mathbb{Z} \oplus \bar{P}_{\mathbb{Z}}$ in $\mathscr{F} \mathscr{A}$.

2. Let $P_{\mathbb{Z}_{2}}$ denote the functor $\mathbb{Z}_{2} \otimes P_{\mathbb{Z}}$ and $\bar{P}_{\mathbb{Z}_{2}}$ the functor $\mathbb{Z}_{2} \otimes \bar{P}_{\mathbb{Z}}$, where $\mathbb{Z}_{2}$ denotes the 2 -adic integers.

By Yoneda, $P_{\mathbb{Z}}$ is projective in $\mathscr{F} \mathscr{A}$, corepresenting evaluation on $\mathbb{F}$. The ring structure of $\mathbb{Z}[V]$ gives a morphism $\mu: P_{\mathbb{Z}} \otimes P_{\mathbb{Z}} \rightarrow P_{\mathbb{Z}}$, which induces $\bar{\mu}: \bar{P}_{\mathbb{Z}} \otimes \bar{P}_{\mathbb{Z}} \rightarrow$ 
$\bar{P}_{\mathbb{Z}}$. There is a reduced diagonal $\bar{\Delta}: \bar{P}_{\mathbb{Z}} \rightarrow \bar{P}_{\mathbb{Z}} \otimes \bar{P}_{\mathbb{Z}}$; by Yoneda, the composition with the projection $P_{\mathbb{Z}} \rightarrow \bar{P}_{\mathbb{Z}}$ is determined by the element $([1]-[0]) \otimes([1]-[0]) \in$ $\left(\bar{P}_{\mathbb{Z}} \otimes \bar{P}_{\mathbb{Z}}\right)(\mathbb{F})$.

Definition 3.2. For $n \in \mathbb{N}$, let $\bar{P}_{\mathbb{Z}}^{n}$ be the image of the iterated product $\bar{\mu}^{(n-1)}$ : $\bar{P}_{\mathbb{Z}}^{\otimes n} \rightarrow \bar{P}_{\mathbb{Z}}$ (respectively $\bar{P}_{\mathbb{Z}_{2}}^{n} \subset \bar{P}_{\mathbb{Z}_{2}}$ ).

The filtration $\cdots \subset \bar{P}_{\mathbb{Z}}^{n+1}(V) \subset \bar{P}_{\mathbb{Z}}^{n}(V) \subset \cdots \subset \bar{P}_{\mathbb{Z}}^{1}(V)=\bar{P}_{\mathbb{Z}}(V)$, for a fixed $V$, has received much attention (see $[\mathbf{P V 7 7}, \mathbf{B V 0 0}]$, for instance). However, these references do not exploit functoriality.

Lemma 3.3.

1. Reduction modulo 2 induces $\left(\bar{P}_{\mathbb{Z}_{2}}\right) / 2 \cong \bar{P}_{\mathbb{F}}$.

2. For $n \in \mathbb{N}$, the surjection $\bar{P}_{\mathbb{Z}_{2}} \rightarrow \bar{P}_{\mathbb{F}}$ induces a commutative diagram in $\mathscr{F} \mathscr{A}$ :

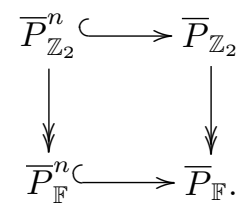

3. The head of $\bar{P}_{\mathbb{Z}_{2}}$ is the functor $\Lambda^{1}$.

Proof. The commutative diagram follows from the morphism of group rings $\mathbb{Z}[V] \rightarrow$ $\mathbb{F}[V]$; the remaining statements are clear.

Lemma 3.4. The composite $\bar{P}_{\mathbb{Z}} \stackrel{\bar{\Delta}}{\rightarrow} \bar{P}_{\mathbb{Z}} \otimes \bar{P}_{\mathbb{Z}} \stackrel{\bar{\mu}}{\rightarrow} \bar{P}_{\mathbb{Z}}$ is the morphism $\bar{P}_{\mathbb{Z}} \stackrel{-2}{\rightarrow} \bar{P}_{\mathbb{Z}}$. Hence, for $n \in \mathbb{N}$, there are inclusions of sub-objects of $\bar{P}_{\mathbb{Z}}: 2 \bar{P}_{\mathbb{Z}}^{n} \subset \bar{P}_{\mathbb{Z}}^{n+1}$ and $2^{n} \bar{P}_{\mathbb{Z}} \subset \bar{P}_{\mathbb{Z}}^{n+1}$.

Proof. The first statement is straightforward (cf. [BV00, Lemma 3.2]); namely, calculating on generators $\bar{\Delta}([v]-[0])=([v]-[0]) \otimes([v]-[0])$ and $\bar{\mu}([v]-[0]) \otimes([w]-[0]))$ $=[v+w]-[v]-[w]+[0]$, so that $\bar{\mu} \bar{\Delta}([v]-[0])=[2 v]-2[v]+[0]=-2([v]-[0])$.

This gives the natural inclusion $2 \bar{P}_{\mathbb{Z}}^{n} \subset \bar{P}_{\mathbb{Z}}^{n+1}$. The final statement follows by induction.

Lemma 3.5. For $n \in \mathbb{N}$, there is a unique non-trivial morphism $\bar{P}_{\mathbb{Z}}^{n} \rightarrow \bar{I}_{\mathbb{F}}$ in $\mathscr{F} \mathscr{A}$, and this induces a surjection $\bar{P}_{\mathbb{Z}}^{n} / \bar{P}_{\mathbb{Z}}^{n+1} \rightarrow p_{n} \bar{I}_{\mathbb{F}}$.

Proof. A morphism $\bar{P}_{\mathbb{Z}}^{n} \rightarrow \bar{I}_{\mathbb{F}}$ factors naturally across $\left(\bar{P}_{\mathbb{Z}}^{n}\right) / 2$. It is straightforward to see that $\left(\left(\bar{P}_{\mathbb{Z}}^{n}\right) / 2\right)(\mathbb{F})=\mathbb{F}$ and $\left(\left(\bar{P}_{\mathbb{Z}}^{n}\right) / 2\right)(0)=0$; this implies that there is a unique non-trivial morphism $\left(\bar{P}_{\mathbb{Z}}^{n}\right) / 2 \rightarrow \bar{I}_{\mathbb{F}}$, by Yoneda.

The composite $\bar{P}_{\mathbb{Z}}^{\otimes n} \rightarrow \bar{P}_{\mathbb{Z}}^{n} \rightarrow \bar{I}_{\mathbb{F}}$ factorizes across the projection $\bar{P}_{\mathbb{Z}}^{\otimes n} \rightarrow \bar{P}_{\mathbb{F}}^{\otimes n}$. Again, there is a unique non-trivial morphism from $\bar{P}_{\mathbb{F}}^{\otimes n}$ to $\bar{I}_{\mathbb{F}}$; for $n=1$, this is the composite $\bar{P}_{\mathbb{F}} \rightarrow \Lambda^{1} \hookrightarrow \bar{I}_{\mathbb{F}}$, and, for $n>1, \bar{P}_{\mathbb{F}}^{\otimes n} \rightarrow \bar{I}_{\mathbb{F}}^{\otimes n} \stackrel{\bar{\mu}^{(n-1)}}{\longrightarrow} \bar{I}_{\mathbb{F}}$, where the first morphism is the iterated tensor product of the morphism $\bar{P}_{\mathbb{F}} \rightarrow \bar{I}_{\mathbb{F}}$. An elementary analysis shows that the composite has image $p_{n} \bar{I}_{\mathbb{F}}$. Hence the morphism $\bar{P}_{\mathbb{Z}}^{n} \rightarrow \bar{I}_{\mathbb{F}}$ has image $p_{n} \bar{I}_{\mathbb{F}}$, by Lemma 3.3 .

Finally, the fact that $\bar{P}_{\mathbb{F}}^{2}$ is the kernel of $\bar{P}_{\mathbb{F}} \rightarrow \Lambda^{1}$ implies that the composite $\bar{P}_{\mathbb{Z}}^{n+1} \hookrightarrow \bar{P}_{\mathbb{Z}}^{n} \rightarrow \bar{I}_{\mathbb{F}}$ is trivial, giving the stated factorization. 
A non-functorial version of the following is contained in [PV77] and [BG10, Section 9.5]; since the functorial version is required here, a direct proof is given.

Proposition 3.6. For $0<n \in \mathbb{Z}$, the following statements hold:

1. There is a short exact sequence $0 \rightarrow 2 \bar{P}_{\mathbb{Z}}^{n} \rightarrow \bar{P}_{\mathbb{Z}}^{n+1} \rightarrow \bar{P}_{\mathbb{F}}^{n+1} \rightarrow 0$.

2. The morphism $\bar{P}_{\mathbb{Z}}^{n} / \bar{P}_{\mathbb{Z}}^{n+1} \rightarrow p_{n} \bar{I}_{\mathbb{F}}$ of Lemma 3.5 is an isomorphism.

Proof. The statements are proved in parallel, by induction upon $n$. For $n=1$, consider the morphism of short exact sequences

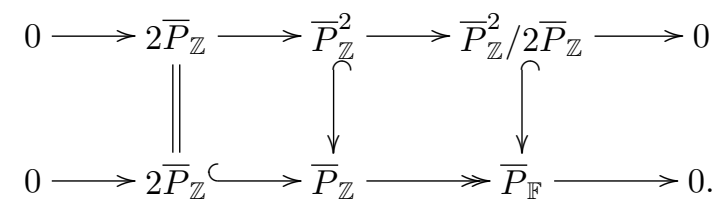

The image of $\bar{P}_{\mathbb{Z}}^{2}$ in $\bar{P}_{\mathbb{F}}$ is $\bar{P}_{\mathbb{F}}^{2}$, by Lemma 3.3 , which proves the first statement; the second follows by studying the cokernels of the monomorphisms.

For the inductive step, suppose the result true for $n<N$. Lemma 3.4 provides a natural inclusion $2^{i} \bar{P}_{\mathbb{Z}} \subset \bar{P}_{\mathbb{Z}}^{i+1}$, for all $i \in \mathbb{N}$; for current purposes, it is sufficient to work with the inclusion $2^{i+1} \bar{P}_{\mathbb{Z}} \subset \bar{P}_{\mathbb{Z}}^{i+1}$. The proof proceeds by providing upper and lower bounds for $\bar{P}_{\mathbb{Z}}^{N+1} / 2^{N+1} \bar{P}_{\mathbb{Z}}$. It is sufficient to do this in the Grothendieck group of $\mathscr{F} \mathscr{A}$, since the functors considered below have only finitely many composition factors of a given isomorphism type, which allows comparison arguments. (Evaluation on finite-dimensional vector spaces would allow reduction to finite objects.)

The element of the Grothendieck group associated to an object $F$ is denoted $[F]$; for the functors considered here, this lies in the submonoid $\prod_{\lambda} \mathbb{N}$ indexed by the isomorphism classes of simple objects $S_{\lambda}$ of $\mathscr{F} \mathscr{A}$. There is a natural partial order: for two objects $M=\Sigma M_{\lambda}\left[S_{\lambda}\right]$ and $N=\Sigma N_{\lambda}\left[S_{\lambda}\right]$, write $M \leqslant N$ if $M_{\lambda} \leqslant N_{\lambda} \forall \lambda$.

The inductive hypothesis implies that $\left[\bar{P}_{\mathbb{Z}}^{N} / 2^{N} \bar{P}_{\mathbb{Z}}\right]=\sum_{i=1}^{N}\left[\bar{P}_{\mathbb{F}}^{i}\right]$ in the Grothendieck group.

The inclusion $2 \bar{P}_{\mathbb{Z}}^{N} \hookrightarrow \bar{P}_{\mathbb{Z}}^{N+1}$ induces a monomorphism

$$
\left(\bar{P}_{\mathbb{Z}}^{N} / 2^{N} \bar{P}_{\mathbb{Z}}\right) \cong 2 \bar{P}_{\mathbb{Z}}^{N} / 2^{N+1} \bar{P}_{\mathbb{Z}} \hookrightarrow \bar{P}_{\mathbb{Z}}^{N+1} / 2^{N+1} \bar{P}_{\mathbb{Z}} ;
$$

the composite with the projection $\bar{P}_{\mathbb{Z}}^{N+1} / 2^{N+1} \bar{P}_{\mathbb{Z}} \rightarrow \bar{P}_{\mathbb{F}}^{N+1}$ is clearly trivial, and hence this gives a lower bound for $\bar{P}_{\mathbb{Z}}^{N+1} / 2^{N+1} \bar{P}_{\mathbb{Z}}$ :

$$
\sum_{i=1}^{N+1}\left[\bar{P}_{\mathbb{F}}^{i}\right] \leqslant\left[\bar{P}_{\mathbb{Z}}^{N+1} / 2^{N+1} \bar{P}_{\mathbb{Z}}\right],
$$

with equality if and only if the first statement holds.

Consider the inclusion $\bar{P}_{\mathbb{Z}}^{N+1} \hookrightarrow \bar{P}_{\mathbb{Z}}^{N}$, which induces the monomorphism

$$
\bar{P}_{\mathbb{Z}}^{N+1} / 2^{N+1} \bar{P}_{\mathbb{Z}} \hookrightarrow \bar{P}_{\mathbb{Z}}^{N} / 2^{N+1} \bar{P}_{\mathbb{Z}}
$$

with cokernel that surjects to $p_{N} \bar{I}_{\mathbb{F}}$, by Lemma 3.5 ; this gives the inequality

$$
\left[\bar{P}_{\mathbb{Z}}^{N} / 2^{N+1} \bar{P}_{\mathbb{Z}}\right] \geqslant\left[\bar{P}_{\mathbb{Z}}^{N+1} / 2^{N+1} \bar{P}_{\mathbb{Z}}\right]+\left[p_{N} \bar{I}_{\mathbb{F}}\right]
$$


with equality if and only if the second statement holds.

The short exact sequence

$$
0 \rightarrow \bar{P}_{\mathbb{F}} \cong\left(2^{N} \bar{P}_{\mathbb{Z}} / 2^{N+1} \bar{P}_{\mathbb{Z}}\right) \rightarrow \bar{P}_{\mathbb{Z}}^{N} / 2^{N+1} \bar{P}_{\mathbb{Z}} \rightarrow \bar{P}_{\mathbb{Z}}^{N} / 2^{N} \bar{P}_{\mathbb{Z}} \rightarrow 0
$$

and the inductive hypothesis give

$$
\left[\bar{P}_{\mathbb{Z}}^{N} / 2^{N+1} \bar{P}_{\mathbb{Z}}\right]=\left[\bar{P}_{\mathbb{F}}\right]+\sum_{i=1}^{N}\left[\bar{P}_{\mathbb{F}}^{i}\right] .
$$

Now $\left[\bar{P}_{\mathbb{F}}\right]=\left[\bar{P}_{\mathbb{F}}^{N+1}\right]+\left[p_{N} \bar{I}_{\mathbb{F}}\right]$ by definition of $q_{N} \bar{P}_{\mathbb{F}}\left(\right.$ since $\left.\left[p_{N} \bar{I}_{\mathbb{F}}\right]=\left[q_{N} \bar{P}_{\mathbb{F}}\right]\right)$; hence (1) implies that

$$
\sum_{i=1}^{N+1}\left[\bar{P}_{\mathbb{F}}^{i}\right] \geqslant\left[\bar{P}_{\mathbb{Z}}^{N+1} / 2^{N+1} \bar{P}_{\mathbb{Z}}\right]
$$

with equality if and only if the second statement holds. Hence both inequalities are equalities and the inductive step is established.

Corollary 3.7. For $V \in \mathrm{Ob} \mathscr{V}^{f}$, the topologies on the abelian group $\bar{P}_{\mathbb{Z}}(V)$ induced by the 2-adic filtration $2^{i} \bar{P}_{\mathbb{Z}}$ and by the filtration $\bar{P}_{\mathbb{Z}}^{i}$ are equivalent.

Proof. By Lemma 3.4, $2^{n} \bar{P}_{\mathbb{Z}} \subset \bar{P}_{\mathbb{Z}}^{n+1}$; conversely, using Proposition 3.6, it is straightforward to show that $\bar{P}_{\mathbb{Z}}^{n+k}\left(\mathbb{F}^{n}\right) \subset 2^{k} \bar{P}_{\mathbb{Z}}\left(\mathbb{F}^{n}\right)$, for $k \in \mathbb{N}$.

\subsection{The structure of the quotients $\bar{P}_{\mathbb{Z}} / \bar{P}_{\mathbb{Z}}^{n+1}$}

Notation 3.8. For $n \in \mathbb{N}$, let $R_{\mathbb{Z}}^{n}$ denote the quotient $\bar{P}_{\mathbb{Z}} / \bar{P}_{\mathbb{Z}}^{n+1}$.

Lemma 3.9. For $0<n \in \mathbb{N}$ :

1. $2^{n+1} R_{\mathbb{Z}}^{n}=0$ and $R_{\mathbb{Z}}^{n}(\mathbb{F}) \cong \mathbb{Z} / 2^{n}$;

2. there are short exact sequences:

$$
\begin{aligned}
& 0 \rightarrow p_{n} \bar{I}_{\mathbb{F}} \rightarrow R_{\mathbb{Z}}^{n} \rightarrow R_{\mathbb{Z}}^{n-1} \rightarrow 0, \\
& 0 \rightarrow R_{\mathbb{Z}}^{n-1} \rightarrow R_{\mathbb{Z}}^{n} \rightarrow q_{n} \bar{P}_{\mathbb{F}} \rightarrow 0 ; \text { and }
\end{aligned}
$$

3. the largest subfunctor ${ }_{2} R_{\mathbb{Z}}^{n}$ of $R_{\mathbb{Z}}^{n}$ annihilated by 2 is isomorphic to $p_{n} \bar{I}_{\mathbb{F}}$.

Proof. The first statement is clear, and the first short exact sequence is provided by Proposition 3.6. The second short exact sequence is induced by the inclusion $2 \bar{P}_{\mathbb{Z}} \hookrightarrow \bar{P}_{\mathbb{Z}}$, since Proposition 3.6 implies that $2 \bar{P}_{\mathbb{Z}} \cap \bar{P}_{\mathbb{Z}}^{n+1}$ is isomorphic to $\bar{P}_{\mathbb{Z}}^{n}$ under the isomorphism $\bar{P}_{\mathbb{Z}} \cong 2 \bar{P}_{\mathbb{Z}}$.

The proof that the largest subfunctor of $R_{\mathbb{Z}}^{n}$ annihilated by 2 is $p_{n} \bar{I}_{\mathbb{F}}$ is by induction on $n$; for $n=1, R_{\mathbb{Z}}^{1} \cong \Lambda^{1}$ and the result is immediate. For the inductive step, the short exact sequence

$$
0 \rightarrow p_{n} \bar{I}_{\mathbb{F}} \rightarrow R_{\mathbb{Z}}^{n} \rightarrow R_{\mathbb{Z}}^{n-1} \rightarrow 0
$$

implies that there is an exact sequence $0 \rightarrow p_{n} \bar{I}_{\mathbb{F}} \rightarrow{ }_{2} R_{\mathbb{Z}}^{n} \rightarrow p_{n-1} \bar{I}_{\mathbb{F}}$, where the righthand term is given by the inductive hypothesis. It suffices to show that the image of ${ }_{2} R_{\mathbb{Z}}^{n}$ in $p_{n-1} \bar{I}_{\mathbb{F}}$ is trivial; hence, by Lemma 2.7 , it suffices to show this after evaluation on $\mathbb{F}$. This follows from the fact that $R_{\mathbb{Z}}^{n}(\mathbb{F}) \cong \mathbb{Z} / 2^{n}$. 
Lemma 3.10. For $0<n \in \mathbb{N}$, the functor $R_{\mathbb{Z}}^{n}$ has simple head $\Lambda^{1}$ and simple socle $\Lambda^{1}$.

Proof. The functor $R_{\mathbb{Z}}^{n}$ is a non-trivial quotient of $\bar{P}_{\mathbb{Z}_{2}}$, which has simple head $\Lambda^{1}$ by Lemma 3.3, and hence $R_{\mathbb{Z}}^{n}$ has simple head $\Lambda^{1}$.

The proof that the socle is $\Lambda^{1}$ is by induction on $n$, starting from the case $n=$ 1 , which is clear, since $R_{\mathbb{Z}}^{1} \cong \Lambda^{1}$ is simple. The exact sequence $0 \rightarrow p_{n} \bar{I}_{\mathbb{F}} \rightarrow R_{\mathbb{Z}}^{n} \rightarrow$ $R_{\mathbb{Z}}^{n-1} \rightarrow 0$ shows that the socle of $R_{\mathbb{Z}}^{n}$ is either $\Lambda^{1}$ or $\Lambda^{1} \oplus \Lambda^{1}$. The latter possibility is excluded since $R_{\mathbb{Z}}^{n}(\mathbb{F})=\mathbb{Z} / 2^{n}$, by Lemma 3.9.

Theorem 3.11. For $0<n \in \mathbb{N}$, the functor $R_{\mathbb{Z}}^{n}$ is self-dual. More precisely, any surjection $\bar{P}_{\mathbb{Z}_{2}} \rightarrow D R_{\mathbb{Z}}^{n}$ factors canonically across an isomorphism $R_{\mathbb{Z}}^{n} \stackrel{\cong}{\rightarrow} D R_{\mathbb{Z}}^{n}$.

Proof. The proof is by induction upon $n$, starting from the case $n=1$, when $R_{\mathbb{Z}}^{n}$ is the simple functor $\Lambda^{1}$, which is self-dual. The factorization statement follows from the fact that $\bar{P}_{\mathbb{Z}_{2}}$ has simple head.

For the inductive step, $R_{\mathbb{Z}}^{n}$ has simple socle $\Lambda^{1}$, hence $D R_{\mathbb{Z}}^{n}$ has simple head $\Lambda^{1}$ and there exists a surjection $\bar{P}_{\mathbb{Z}_{2}} \rightarrow D R_{\mathbb{Z}}^{n}$ (by projectivity of $\bar{P}_{\mathbb{Z}}$ ). This gives rise to a morphism of short exact sequences

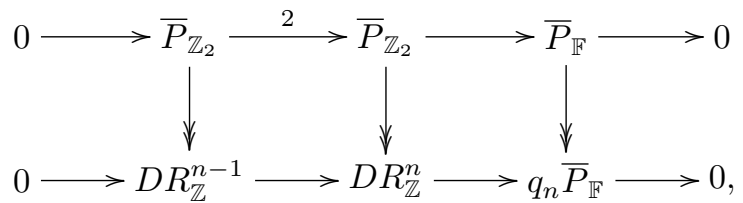

where the lower exact sequence is the dual of the first exact sequence of Lemma 3.9, the commutativity of the right-hand square follows from the fact that there is a unique non-trivial morphism $\bar{P}_{\mathbb{Z}_{2}} \rightarrow q_{n} \bar{P}_{\mathbb{F}}$, and the surjectivity of the left-hand vertical morphism is seen by evaluating on $\mathbb{F}$, since $D R_{\mathbb{Z}}^{n-1}$ has simple head.

By the inductive hypothesis, the left-hand vertical morphism factorizes across an isomorphism $R_{\mathbb{Z}}^{n-1} \stackrel{\cong}{\rightarrow} D R_{\mathbb{Z}}^{n-1}$. In particular, this implies that $\bar{P}_{\mathbb{Z}_{2}}^{n+1}$ lies in the kernel of $\bar{P}_{\mathbb{Z}_{2}} \rightarrow D R_{\mathbb{Z}}^{n}$; hence this induces a surjection $R_{\mathbb{Z}}^{n} \rightarrow D R_{\mathbb{Z}}^{n}$, which is an isomorphism, since the objects have finite composition series with isomorphic associated graded functors.

Definition 3.12. Let $R_{\mathbb{Z}}^{\infty}$ be the direct $\operatorname{limit} \lim _{\rightarrow} R_{\mathbb{Z}}^{n}$ of the diagram of monomorphisms provided by Lemma 3.9.

Theorem 3.13. There are Pontrjagin duality isomorphisms: $R_{\mathbb{Z}}^{\infty} \cong D \bar{P}_{\mathbb{Z}_{2}}$ and $\bar{P}_{\mathbb{Z}_{2}} \cong$ $D R_{\mathbb{Z}}^{\infty}$.

Proof. The functor $R_{\mathbb{Z}}^{\infty}$ takes values in torsion 2-groups. By construction and Corollary 3.7, the functor $\bar{P}_{\mathbb{Z}_{2}}$ is isomorphic to $\lim _{\leftarrow} R_{\mathbb{Z}}^{n}$. Applying the Pontrjagin duality functor and using the fact that each $R_{\mathbb{Z}}^{n}$ is self dual gives a direct system that is isomorphic to that defining $R_{\mathbb{Z}}^{\infty}$ (cf. proof of Theorem 3.11). The result follows from Pontrjagin duality for abelian groups.

\section{Milnor derivations}

Throughout this section, $p=2$. 


\subsection{Milnor derivations on symmetric powers}

Notation 4.1. For $i \in \mathbb{N}$, let $Q_{i}: S^{1} \rightarrow S^{2^{i+1}}$ denote both the iterated Frobenius $x \mapsto$ $x^{2^{i+1}}$ and its extension $S^{n} \stackrel{Q_{i}}{\rightarrow} S^{n+2^{i+1}-1}$ to a derivation of $S^{*}$.

The following is standard:

Lemma 4.2. For $i, j \in \mathbb{N}, Q_{i} \circ Q_{i}=0$ and the derivations $Q_{i}, Q_{j}$ commute.

Notation 4.3. For $0<j \in \mathbb{N}$, let $S^{*} /\left\langle x^{2^{j}}\right\rangle$ denote the truncated symmetric power functor.

In the following statement, the degree is inherited from $S^{*}$.

Proposition 4.4. For $i \in \mathbb{N}$, the homology $H\left(S^{*}, Q_{i}\right)$ is the truncated symmetric power functor $S^{*} /\left\langle x^{2^{i}}\right\rangle$, concentrated in even degrees. Explicitly, the degree-doubling Frobenius induces the isomorphism:

$$
H\left(S^{*}, Q_{i}\right)^{k} \cong \begin{cases}0 & k \text { odd } \\ S^{d} /\left\langle x^{2^{i}}\right\rangle & k=2 d .\end{cases}
$$

Proof. Non-functorially, the result follows from the calculation of the homology of the differential graded algebra $\left(\mathbb{F}[x], d x=x^{2^{i+1}}\right)$, which is the truncated polynomial algebra $\mathbb{F}[y] / y^{2^{i}}$, where $y=x^{2}$, using the Künneth theorem to extend to tensor products.

It remains to show that this corresponds to the stated functorial isomorphism. By the above, the homology is concentrated in even degrees; moreover, in degree $k=2 d$, the Frobenius $S^{d} \hookrightarrow S^{2 d}$ maps to the cycles in degree $k$ and induces a surjection onto the homology. The identification of the kernel is straightforward.

Remark 4.5. For $i=0$, the homology is $\mathbb{F}$ concentrated in degree 0 ; in particular, the operations $Q_{0}$ induce an exact complex $0 \rightarrow S^{1} \rightarrow S^{2} \rightarrow S^{3} \rightarrow \cdots$.

\subsection{The $Q_{0}$-kernel complex}

Notation 4.6. For $n \in \mathbb{N}$, let $K_{n}$ denote the kernel of $Q_{0}: S^{n} \rightarrow S^{n+1}$.

Lemma 4.7. The derivation $Q_{1}$ on $S^{*}$ induces a differential $Q_{1}: K_{n} \rightarrow K_{n+3}$ and there is a short exact sequence of complexes

$$
0 \rightarrow\left(K_{i+3 \bullet}, Q_{1}\right) \rightarrow\left(S^{i+3 \bullet}, Q_{1}\right) \rightarrow\left(K_{i+1+3 \bullet}, Q_{1}\right) \rightarrow 0,
$$

where $0 \leqslant i<3$ and $\bullet \geqslant 0$.

Proof. A consequence of the exactness of the $Q_{0}$-complex in positive dimensions and the commutation of $Q_{0}, Q_{1}$ (Lemma 4.2).

Notation 4.8. For $n \in \mathbb{N}$, let $L_{n}$ denote the image of $Q_{1}: K_{n-3} \rightarrow K_{n}$, and let $\tilde{L}_{n}$ denote the kernel of $Q_{1}: K_{n} \rightarrow K_{n+3}$.

Proposition 4.9. The homology of the complexes $\left(K_{i+3}, Q_{1}\right)$ is

$$
\tilde{L}_{n} / L_{n} \cong \begin{cases}\mathbb{F} & n=0 \\ 0 & n \text { odd } \\ p_{d} \bar{I}_{\mathbb{F}} & n=2 d>0 .\end{cases}
$$


Proof. The proof is by induction upon $n$; the case $n=0$ is clear. It is also straightforward to show that the odd degree homology is trivial (independently of the calculation of the even degree homology).

For the inductive step, the homology long exact sequence arising from the short exact sequence of complexes of Lemma 4.7 is used. This shows that, for $n=2 d>0$, the homology $H$ of $K_{n-3} \rightarrow K_{n} \rightarrow K_{n+3}$ lies in a short exact sequence $0 \rightarrow p_{d-1} \bar{I}_{\mathbb{F}} \rightarrow$ $H \rightarrow \Lambda^{d} \rightarrow 0$, by the inductive hypothesis for the left-hand term and Proposition 4.4 for the exterior power, using the vanishing of homology in odd degrees. It suffices to show that $p_{d} \bar{I}_{\mathbb{F}}$ is a subquotient of $H$, by Lemma 2.6 .

The Frobenius $\Phi: S^{d} \hookrightarrow S^{2 d}$ maps to $K_{2 d}$, and the image lies in the kernel $\tilde{L}_{2 d}$ of $K_{2 d} \rightarrow K_{2 d+3}$. There is a unique non-trivial morphism $S^{d} \rightarrow \bar{I}_{\mathbb{F}}$, and this has image $p_{d} \bar{I}_{\mathbb{F}}$; since $\bar{I}_{\mathbb{F}}$ is injective, this extends to a morphism $\tilde{L}_{2 d} \rightarrow \bar{I}_{\mathbb{F}}$, and the restriction $K_{2 d-3} \rightarrow \bar{I}_{\mathbb{F}}$ is trivial, since $K_{2 d-3}(\mathbb{F})=0$. It follows that $p_{d} \bar{I}_{\mathbb{F}}$ is a subquotient of the homology $H$, as required.

\section{Connective complex K-cohomology and homology}

\subsection{Recollections}

The Postnikov towers of $k u$ and $K U$ provide morphisms $k u \rightarrow H \mathbb{Z}$ and $k u \rightarrow K U$ of commutative ring spectra, relating connective (resp. periodic) complex $K$-theory $k u$ (resp. $K U$ ) and the integral Eilenberg-MacLane spectrum $H \mathbb{Z}$. There are cofibre sequences $\Sigma^{2} k u \stackrel{v}{\rightarrow} k u \rightarrow H \mathbb{Z}, H \mathbb{Z} \stackrel{2}{\rightarrow} H \mathbb{Z} \stackrel{\rho}{\rightarrow} H \mathbb{F}$, where $v$ is multiplication by the Bott element $\left(k u_{*} \cong \mathbb{Z}[v]\right.$, with $\left.|v|=2\right)$.

Notation 5.1. Let $\mathfrak{Q}$ denote the first $k$-invariant of $k u$, given by the composite $H \mathbb{Z} \rightarrow$ $\Sigma^{3} k u \rightarrow \Sigma^{3} H \mathbb{Z}$.

The Milnor derivation $Q_{0}$ of Section 4 identifies with the Bockstein $\beta=S q^{1}$ and the Milnor derivation $Q_{1}$ with the commutator $\left[S q^{2}, S q^{1}\right]$.

Lemma 5.2. (cf. [Ada74, proof of III.16.6]) There is a commutative diagram

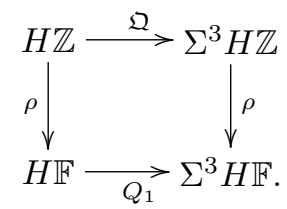

Lemma 5.3. For $Y$ a spectrum, the following conditions are equivalent:

1. $H \mathbb{Z}^{*} Y \stackrel{\rho}{\rightarrow} H \mathbb{F}^{*} Y$ is a monomorphism.

2. The Bockstein complex $\left(H \mathbb{F}^{*} Y, \beta\right)$ is exact.

3. $H \mathbb{Z}_{*} Y \stackrel{\rho}{\rightarrow} H \mathbb{F}_{*} Y$ is a monomorphism.

4. The Bockstein complex $\left(H \mathbb{F}_{*} Y, \beta\right)$ is exact.

When these conditions are satisfied, the respective morphisms $\mathfrak{Q}$ are determined by 
the commutative diagrams
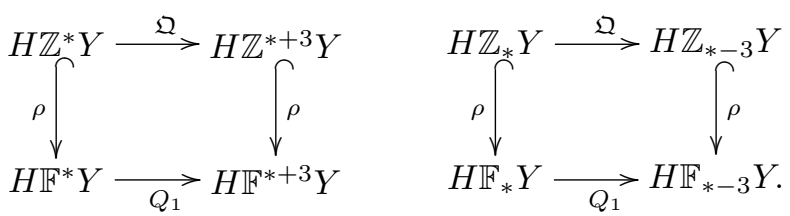

Proof. Straightforward, applying Lemma 5.2 for the commutative diagrams.

Example 5.4. The hypotheses of Lemma 5.3 are satisfied for $Y=\Sigma^{\infty} B V$, where $V$ is an elementary abelian 2-group. In particular, the action of $\mathfrak{Q}$ on $H \mathbb{Z}^{*}(B V)$ is determined by the action of $\Lambda\left(Q_{0}, Q_{1}\right)$ on $H \mathbb{F}^{*}(B V)$.

\subsection{On $v$-torsion and cotorsion}

There is a hereditary torsion theory on the category of graded $\mathbb{Z}[v]$-modules (taking $|v|=2$ ) given by the categories of $v$-power torsion modules and of $v$-cotorsion modules. This has associated torsion functor tors $v_{v}$ and cotorsion functor $\operatorname{cotors}_{v}$ so that, for a $\mathbb{Z}[v]$-module, there is a natural short exact sequence of $\mathbb{Z}[v]$-modules $0 \rightarrow \operatorname{tors}_{v} M \rightarrow M \rightarrow \operatorname{cotors}_{v} M \rightarrow 0$. The submodule of $M$ annihilated by $v$ is written $\operatorname{ann}_{v} M$.

Lemma 5.5. For a $\mathbb{Z}[v]$-module $M$, $\operatorname{tors}_{v} M \cap v M \cong v \operatorname{tors}_{v} M$; hence $\operatorname{tors}_{v} M \hookrightarrow M$ induces a monomorphism $\left(\operatorname{tors}_{v} M\right) / v \hookrightarrow M / v$. Moreover, there is a short exact sequence of $\mathbb{Z}[v]$-modules

$$
0 \rightarrow \operatorname{cotors}_{v} M \stackrel{v}{\rightarrow} \operatorname{cotors}_{v} M \rightarrow(M / v) /\left(\left(\operatorname{tors}_{v} M\right) / v\right) \rightarrow 0 .
$$

Proof. Straightforward.

The following algebraic result is required in the proof of Proposition 5.9.

Lemma 5.6. For $M$ a graded $\mathbb{Z}[v]$-module, the following conditions are equivalent:

1. $\operatorname{ann}_{v} M=\operatorname{tors}_{v} M$ and

2. $\mathbf{a n n}_{v} M \rightarrow\left(\operatorname{tors}_{v} M\right) / v$ is injective, and they imply

3. $\operatorname{ann}_{v} M \rightarrow\left(\operatorname{tors}_{v} M\right) / v$ is surjective.

If $\forall d \in \mathbb{Z} \exists N(d) \in \mathbb{N}$ such that $\left(v^{N(d)} M \cap \text { tors }_{v} M\right)^{d}=0$, then the conditions are all equivalent.

Proof. If $\mathbf{a n n}_{v} M=\operatorname{tors}_{v} M$, then $\operatorname{tors}_{v} M \cong\left(\operatorname{tors}_{v} M\right) / v$, and hence condition (1) implies both (2) and (3).

Suppose (2) holds, and consider $x \in \operatorname{tors}_{v} M$, so that there exists $t \in \mathbb{N}$ such that $v^{t} x \neq 0$ and $v^{t+1} x=0$. Hence $v^{t} x \in \mathbf{a n n}_{v} M$; the hypothesis implies that $v^{t} x$ is not in $v M$; hence $t=0$ and $x \in \mathbf{a n n}_{v} M$, as required.

Suppose (3) holds together with the additional hypothesis. Consider $x \in \operatorname{tors}_{v} M$; by surjectivity of $\mathbf{a n n}_{v} M \rightarrow\left(\operatorname{tors}_{v} M\right) / v, x=a+v y$, for some $a \in \mathbf{a n n}_{v} M$ and $y \in$ $\operatorname{tors}_{v} M$. An induction then shows that, for $0<n \in \mathbb{N}, x=a+v^{n} y_{n}$, for some $y_{n} \in$ $\operatorname{tors}_{v} M$; it suffices to show that $v^{n} y_{n}=0$ for $n \gg 0$. By construction $v^{n} y_{n} \in\left(v^{n} M \cap\right.$ 
$\left.\operatorname{tors}_{v} M\right)^{|x|}$, and hence the hypothesis implies that the element is zero for $n \geqslant N(|x|)$.

The following allows the cotorsion to be related to periodic $K$-theory.

Lemma 5.7. [BG03, Chapter 1] If $Z$ is a connective spectrum, there is a natural isomorphism $K U^{*}(Z) \cong k u^{*}(Z)\left[\frac{1}{v}\right]$.

Lemma 5.8. For $X$ a spectrum, there are natural short exact sequences

$$
\begin{gathered}
0 \rightarrow k u_{*}(X) / v \rightarrow H \mathbb{Z}_{*}(X) \rightarrow \mathbf{a n n}_{v} k u_{*-3}(X) \rightarrow 0, \\
0 \rightarrow k u^{*}(X) / v \rightarrow H \mathbb{Z}^{*}(X) \rightarrow \mathbf{a n n}_{v} k u^{*+3}(X) \rightarrow 0 .
\end{gathered}
$$

Moreover, there are natural inclusions

$$
\begin{gathered}
\operatorname{Im} \mathfrak{Q} \subset\left(\operatorname{tors}_{v} k u_{*}(X)\right) / v \subset k u_{*}(X) / v \subset \operatorname{Ker} \mathfrak{Q}, \\
\operatorname{Im} \mathfrak{Q} \subset\left(\operatorname{tors}_{v} k u^{*}(X)\right) / v \subset k u^{*}(X) / v \subset \operatorname{Ker} \mathfrak{Q} .
\end{gathered}
$$

Proof. The proofs for homology and cohomology are formally the same; hence consider $k u$-homology. The short exact sequence is induced by the cofibre sequence $\Sigma^{2} k u \rightarrow k u \rightarrow H \mathbb{Z}$. The inclusion $\left(\operatorname{tors}_{v} k u_{*}(X)\right) / v \subset k u_{*}(X) / v$ is provided by Lemma 5.5; the outer inclusions are clear, from the definition of $\mathfrak{Q}$.

Proposition 5.9. Let $Z$ be a connective spectrum.

1. The following conditions are equivalent:

(a) $\operatorname{ann}_{v} k u_{*}(Z)=$ tors $_{v} k u_{*}(Z)$.

(b) $\left(\operatorname{tors}_{v} k u_{*}(Z)\right) / v \cong \operatorname{ImQ}$.

(c) $k u_{*}(Z) / v \cong \operatorname{KerQ}$.

If these conditions are satisfied, then $\left(k u_{*}(Z) / v\right) /\left(\left(\operatorname{tors}_{v} k u_{*}(Z)\right) / v\right) \cong$ $\mathrm{KerQ} / \operatorname{Im} \mathfrak{Q}$ and there is a short exact sequence of $\mathbb{Z}[v]$-modules

$$
0 \rightarrow \operatorname{cotors}_{v} k u_{*-2}(Z) \stackrel{v}{\rightarrow} \operatorname{cotors}_{v} k u_{*}(Z) \rightarrow \operatorname{KerQ} / \operatorname{ImQ} \rightarrow 0
$$

and a pullback of short exact sequences

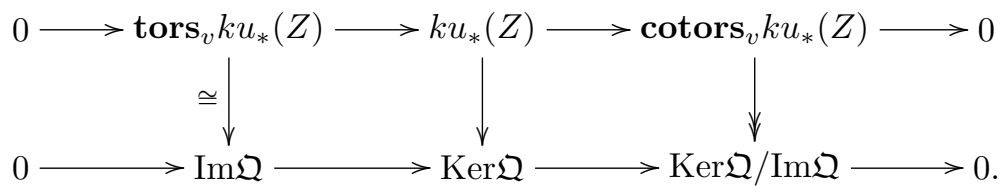

2. Suppose that, for each degree $d \in \mathbb{Z}$, there exists an integer $N(d)$ such that $\left(v^{N(d)} k u^{*}(Z) \cap \operatorname{tors}_{v} k u^{*}(Z)\right)^{d}=0$; then the following conditions are equivalent:

(a) $\operatorname{ann}_{v} k u^{*}(Z)=\operatorname{tors}_{v} k u^{*}(Z)$.

(b) $\left(\operatorname{tors}_{v} k u^{*}(Z)\right) / v \cong \operatorname{ImQ}$.

(c) $k u^{*}(Z) / v \cong \operatorname{KerQ}$. 
If these conditions are satisfied, then $\left(k u^{*}(Z) / v\right) /\left(\left(\operatorname{tors}_{v} k u^{*}(Z)\right) / v\right) \cong$ $\mathrm{KerQ} / \operatorname{ImQ}$ and there is a short exact sequence of $\mathbb{Z}[v]$-modules

$$
0 \rightarrow \operatorname{cotors}_{v} k u^{*+2}(Z) \stackrel{v}{\rightarrow} \operatorname{cotors}_{v} k u^{*}(Z) \rightarrow \operatorname{KerQ} / \operatorname{ImQ} \rightarrow 0
$$

and a pullback of short exact sequences

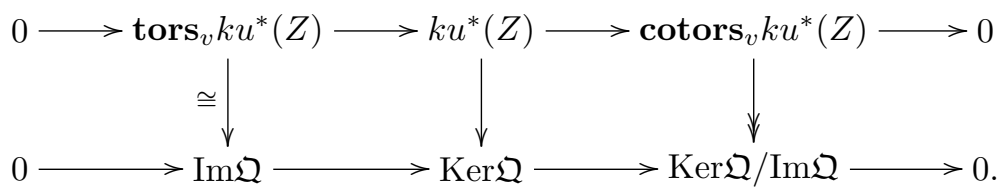

Proof. The hypotheses imply that, for $M \in\left\{k u_{*}(Z), k u^{*}(Z)\right\}$, the three conditions of Lemma 5.6 are equivalent. The equivalence of conditions (a), (b), and (c) follows from an analysis of the short exact sequences of Lemma 5.8. Consider $k u$-homology (the argument for $k u$-cohomology is similar); there is a commutative diagram

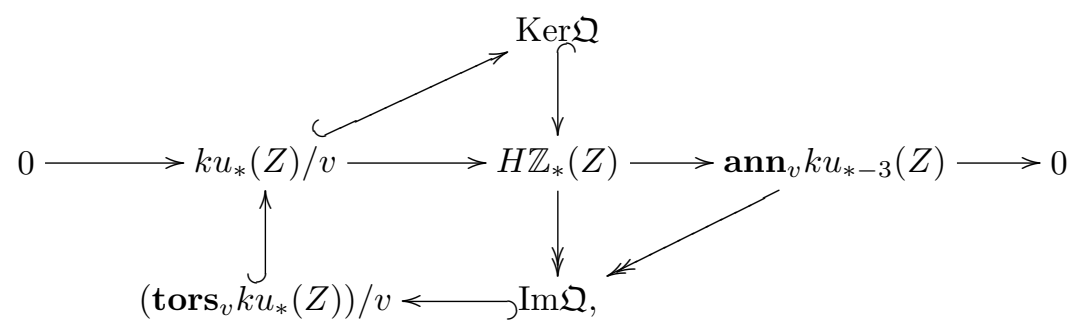

in which the middle row and column are short exact.

By the five-lemma, $\operatorname{Im} \mathfrak{Q} \cong \mathbf{a n n}_{v} k u_{*-3}(Z)$ if and only if $\operatorname{Ker} \mathfrak{Q} \cong k u_{*}(Z) / v$. Moreover, Lemma 5.6 implies that the following three conditions are equivalent:

1. $\operatorname{ann}_{v} k u_{*}(Z)=\operatorname{tors}_{v} k u_{*}(Z)$.

2. $\operatorname{ImQ} \cong\left(\operatorname{tors}_{v} k u_{*}(Z)\right) / v$.

3. $\operatorname{ImQ} \cong \mathbf{a n n}_{v} k u_{*-3}(Z)$.

This shows that conditions (a), (b), and (c) are equivalent. The consequences follow, using Lemma 5.5 to provide the short exact sequence for $\operatorname{cotors}_{v} k u_{*}(Z) / v$.

The nilpotency hypothesis of Proposition 5.9 is supplied by the following result, which provides a uniform bound on nilpotency.

Lemma 5.10. [BG03, Lemma 1.5.8] Let $Y$ be a space such that $k u^{*}\left(Y_{+}\right)$is a Noetherian $\mathbb{Z}[v]$-algebra. Then $\exists N \in \mathbb{N}$ such that $v^{N} \operatorname{tors}_{v} k u^{*}\left(Y_{+}\right)=0$.

This applies to the classifying space of a finite group $G$, since $k u^{*}\left(B G_{+}\right)$is a Noetherian $\mathbb{Z}[v]$-algebra (see, for example, [BG03, Section 1.1]).

\subsection{The $k u$-cohomology of $B V_{+}$}

The above methods apply in considering the $k u$-cohomology $k u^{*}\left(B V_{+}\right)$of an elementary abelian 2-group. By the Atiyah-Segal completion theorem, the periodic $K$ theory $K U^{*}\left(B V_{+}\right)$is trivial in odd degrees and isomorphic in even degrees to the completion at the augmentation ideal of the complex representation ring. 
Proposition 5.11. There are natural isomorphisms

$$
\begin{aligned}
H \mathbb{Z}^{n}\left(B V_{+}\right) & \cong \begin{cases}\mathbb{Z} & n=0 \\
K_{n}\left(V^{\sharp}\right) & n>0\end{cases} \\
(\operatorname{Im} \mathfrak{Q})^{n} & \cong L_{n}\left(V^{\sharp}\right) \\
(\operatorname{Ker} \mathfrak{Q})^{n} & \cong \begin{cases}\mathbb{Z} & n=0 \\
\tilde{L}_{n}\left(V^{\sharp}\right) & n>0 .\end{cases}
\end{aligned}
$$

Proof. The algebra $H \mathbb{F}^{*}\left(B V_{+}\right)$is naturally isomorphic to $S^{*}\left(V^{\sharp}\right)$ and integral reduced cohomology $H \mathbb{Z}^{*}(B V)$ embeds in $H \mathbb{F}^{*}(B V)$ as the kernel of the Bockstein operator. The result follows from Lemmas 5.2 and 5.3, using the definition of the functors $K_{n}$, $L_{n}$ and $\tilde{L}_{n}$ from Section 4 .

Lemma 5.12. (cf. [BG03, Section 2.2]) There are identities $\operatorname{tors}_{v} k u^{*}\left(B 0_{+}\right)=0=$ $\operatorname{tors}_{v} k u^{*}\left(B \mathbb{Z} / 2_{+}\right)$.

Theorem 5.13. For $V \in \mathrm{Ob} \mathscr{V}^{f}$, there are natural isomorphisms

$$
\begin{aligned}
\operatorname{tors}_{v} k u^{n}\left(B V_{+}\right) & \cong \mathbf{a n n}_{v} k u^{n}\left(B V_{+}\right) \cong(\operatorname{ImQ})^{n} \\
k u^{n}\left(B V_{+}\right) / v & \cong(\operatorname{Ker} \mathfrak{Q})^{n} ;
\end{aligned}
$$

$\operatorname{tors}_{v} k u^{n}\left(B V_{+}\right) \rightarrow k u^{n}\left(B V_{+}\right) / v$ is an isomorphism for $n$ odd, and, for $n=2 d>0$, there is a natural short exact sequence

$$
0 \rightarrow \operatorname{tors}_{v} k u^{2 d}(B V) \rightarrow k u^{2 d}(B V) / v \rightarrow p_{d} \bar{I}_{\mathbb{F}}\left(V^{\sharp}\right) \rightarrow 0 .
$$

There is a pullback of short exact sequences:

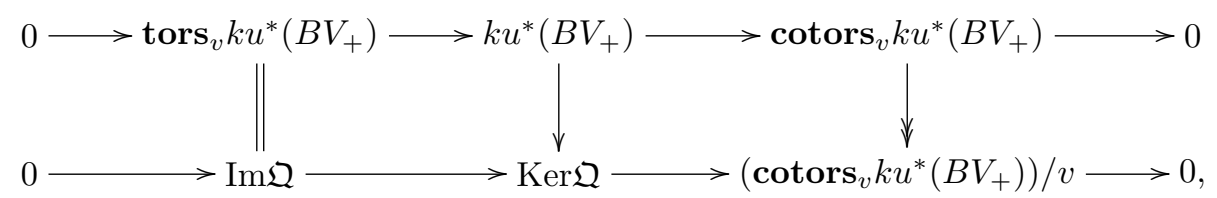

where, for $n \in \mathbb{N}, \operatorname{cotors}_{v} k u^{n}\left(B V_{+}\right) \cong \begin{cases}\mathbb{Z} \oplus \bar{P}_{\mathbb{Z}_{2}}^{d}\left(V^{\sharp}\right) & n=2 d \\ 0 & \text { otherwise. }\end{cases}$

Proof. The first part of the Theorem follows from Proposition 5.9, since Lemma 2.7 implies that the cohomological hypothesis is satisfied. To apply the proposition, it is sufficient to show that $\operatorname{ImQ} \cong\left(\operatorname{tors}_{v} k u^{*}\left(B V_{+}\right)\right) / v$.

By Proposition 5.11 and Proposition 4.9,

$$
(\operatorname{KerQ} / \operatorname{Im} \mathfrak{Q})^{n} \cong \begin{cases}\mathbb{Z} & n=0 \\ 0 & n \text { odd } \\ p_{d} \bar{I}_{\mathbb{F}}\left(V^{\sharp}\right) & n=2 d, d>0 .\end{cases}
$$

In odd degrees, $\operatorname{Im} \mathfrak{Q}=\operatorname{Ker} \mathfrak{Q}$, and the result follows from the inclusions given in Lemma 5.8.

It remains to show that the inclusion $(\operatorname{ImQ})^{2 d} \hookrightarrow \operatorname{tors}_{v} k u^{2 d}\left(B V_{+}\right) / v$ is an isomorphism for $d \in \mathbb{N}$. For $d=0$, both terms are zero; for $d>0$, the cokernel is a subfunctor of $V \mapsto p_{d} \bar{I}_{\mathbb{F}}\left(V^{\sharp}\right)$ by the inclusions given in Lemma 5.8 and the above identification of KerQ/ImQ; hence it suffices to show that the cokernel is trivial when evaluated 
on $V=\mathbb{F}$, by Lemma 2.7. This follows from the fact that $\operatorname{tors}_{v} k u^{*}\left(B \mathbb{Z} / 2_{+}\right)=0$, by Lemma 5.12.

Finally, consider $\operatorname{cotors}_{v} k u^{*}\left(B V_{+}\right)$. For $d=0$, the result is the Atiyah-Segal completion theorem; the structure in higher degrees follows by induction on $d$ from the results of Section 3.1, in particular Proposition 3.6, using the identification of the functors $\left(\operatorname{cotors}_{v} k u^{*}\left(B V_{+}\right)\right) / v \cong \operatorname{KerQ} / \operatorname{ImQ}$ given above.

Remark 5.14. The above result determines the functor $V \mapsto k u^{*}\left(B V_{+}\right)$as a functor to graded $\mathbb{Z}[v]$-modules. Moreover, the multiplicative structure can be deduced from the natural monomorphism $k u^{*}\left(B V_{+}\right) \hookrightarrow H \mathbb{Z}^{*}\left(B V_{+}\right) \prod K U^{*}\left(B V_{+}\right)$(cf. [BG03]).

\subsection{The $k u$-homology of elementary abelian 2-groups}

The $k u$-homology of elementary abelian 2-groups can be determined as for $k u$ cohomology, by applying Proposition 5.9. The following is dual to Proposition 5.11.

Proposition 5.15. There are natural isomorphisms

$$
\begin{aligned}
H \mathbb{Z}_{n}\left(B V_{+}\right) & \cong \begin{cases}\mathbb{Z} & n=0 \\
D K_{n+1}(V) & n>0\end{cases} \\
(\operatorname{Im} \mathfrak{Q})_{n} & \cong D L_{n+4} \\
(\operatorname{Ker} \mathfrak{Q})_{n} & \cong \begin{cases}\mathbb{Z} & n=0 \\
D\left(K_{n+1} / L_{n+1}\right)(V) & n>0 .\end{cases}
\end{aligned}
$$

Moreover

$$
(\operatorname{KerQ} / \operatorname{Im} \mathfrak{Q})_{n} \cong \begin{cases}\mathbb{Z} & n=0 \\ 0 & n \equiv 0 \quad \bmod 2 \\ q_{d} \bar{P}_{\mathbb{F}}(V) & n=2 d-1>0 .\end{cases}
$$

Theorem 5.16. For $V \in \mathrm{Ob} \mathscr{V}^{f}$, there are natural isomorphisms

$$
\begin{aligned}
\operatorname{tors}_{v} k u_{n}\left(B V_{+}\right) & \cong \mathbf{a n n}_{v} k u_{n}\left(B V_{+}\right) \cong(\operatorname{ImQ})_{n} \\
k u_{n}\left(B V_{+}\right) / v & \cong(\operatorname{KerQ})_{n} ;
\end{aligned}
$$

the inclusion $\operatorname{tors}_{v} k u_{*}\left(B V_{+}\right) \hookrightarrow k u_{*}\left(B V_{+}\right)$induces a natural short exact sequence

$$
0 \rightarrow \operatorname{tors}_{v} k u_{*}\left(B V_{+}\right) \rightarrow k u_{*}\left(B V_{+}\right) / v \rightarrow \operatorname{cotors}_{v} k u_{*}\left(B V_{+}\right) / v \rightarrow 0,
$$

where

$$
\operatorname{cotors}_{v} k u_{n}\left(B V_{+}\right) / v \cong \begin{cases}\mathbb{Z} & n=0 \\ 0 & 0<n \equiv 0 \bmod 2 \\ q_{d} \bar{P}_{\mathbb{F}}(V) & n=2 d-1>0 .\end{cases}
$$

There is a pullback diagram of short exact sequences

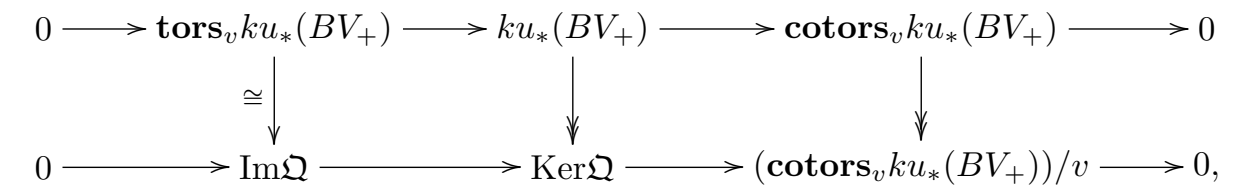

where, for $n \in \mathbb{N}, \operatorname{cotors}_{v} k u(B V) \cong \begin{cases}\mathbb{Z} & n \text { even } \\ R_{\mathbb{Z}}^{d}(V) & n=2 d-1 .\end{cases}$ 
Proof. There are natural monomorphisms $\operatorname{Im} \mathfrak{Q}(V) \hookrightarrow k u_{*}\left(B V_{+}\right) / v \hookrightarrow \operatorname{Ker} \mathfrak{Q}(V)$. By Proposition 5.15, in positive even degree, these are isomorphisms; in degree $n=2 d-$ $1>0$, the quotient $(\operatorname{KerQ} / \operatorname{ImQ})(V)$ is $q_{d} \bar{P}_{\mathbb{F}}(V)$, and hence there is a natural inclusion

$$
\left(\left(k u_{*}\left(B V_{+}\right) / v\right) / \operatorname{ImQ}(V)\right)_{2 d-1} \hookrightarrow q_{d} \bar{P}_{\mathbb{F}}(V) .
$$

To prove the result, by Proposition 5.9, it suffices to show that this is an isomorphism; hence, by Lemma 2.7, it suffices to show that the left-hand side is non-trivial when evaluated on $\mathbb{F}$, for all $d>0$. It is straightforward to verify that $\operatorname{Im} \mathfrak{Q}_{\text {odd }}(\mathbb{F})=0$; thus it suffices to show that $k u_{*}\left(B \mathbb{Z} / 2_{+}\right) / v$ is non-trivial in all odd degrees, which follows from the structure of $k u_{*}\left(B \mathbb{Z} / 2_{+}\right)$as a graded abelian group (see [BG03, Section 3.4], for example).

Finally, the identification of $\operatorname{cotors}_{v} k u_{*}\left(B V_{+}\right)$follows from the results of Section 3.2, in particular the short exact sequences of Lemma 3.9, the self-duality of the functors $R_{\mathbb{Z}}^{n}$ (Theorem 3.11), and the Pontrjagin duality between $R_{\mathbb{Z}}^{\infty}$ and $\bar{P}_{\mathbb{Z}_{2}}$ (Theorem 3.13).

Remark 5.17. By [BG03, Proposition 3.2.1], the universal coefficient spectral sequence calculating $k u^{*}(B V)$ from $k u_{*}(B V)$ collapses to the short exact sequence

$$
\operatorname{Ext}_{k u_{*}}^{2}\left(\Sigma^{2} \operatorname{tors}_{v} k u_{*}(B V), k u_{*}\right) \rightarrow k u^{*}(B V) \rightarrow \operatorname{Ext}_{k u_{*}}^{1}\left(\Sigma^{1} \operatorname{cotors}_{v} k u_{*}(B V), k u_{*}\right) .
$$

This is isomorphic to $0 \rightarrow \operatorname{tors}_{v} k u^{*}(B V) \rightarrow k u^{*}(B V) \rightarrow \operatorname{cotors}_{v} k u^{*}(B V) \rightarrow 0$, and explains the duality between $k u$-homology and $k u$-cohomology of $B V$. The analysis of [BG03, Section 4.12] can be made functorial to give the identification of $\operatorname{cotors}_{v} k u_{*}\left(B V_{+}\right)$.

\section{Filtering symmetric powers and Koszul complexes}

To calculate the local cohomology of $\operatorname{tors}_{v} k u^{*}\left(B V_{+}\right)$at the prime 2 by using a form of local duality (see Section 7), it is necessary to filter and study the associated Koszul complexes, refining the results of Section 4.

The symmetric power functors $S^{*}$ are considered as taking values in graded commutative $\mathbb{F}$-algebras.

\subsection{Filtering the symmetric powers}

Let $\Phi S^{*}$ denote the image of the Frobenius $x \mapsto x^{2}$, so that $\Phi S^{*}$ takes values in graded commutative algebras and the canonical morphisms $S^{*} \cong$ morphisms of graded algebras.

Definition 6.1. For $t \in \mathbb{N}$, let $f_{t} S^{*} \subset S^{*}$ denote the image of $S^{t} \otimes \Phi S^{*} \stackrel{\mu}{\rightarrow} S^{*}$.

Lemma 6.2. For $t \in \mathbb{N}$ :

1. The functors $f_{t} S^{*} \subset S^{*}$ define an increasing filtration of $S^{*}$

$$
f_{0} S^{*}=\Phi S^{*} \subset f_{1} S^{*} \subset f_{2} S^{*} \subset \cdots \subset f_{t} S^{*} \subset \cdots \subset S^{*}
$$

in $\Phi S^{*}$-modules.

2. There is an isomorphism of $S^{*}$-modules $f_{t} S^{*} / f_{t-1} S^{*} \cong \Lambda^{t} \otimes S^{*}$, where $S^{*}$ acts on the left-hand side by restriction along $S^{*} \stackrel{\Phi \cong}{\rightrightarrows} \Phi S^{*}$ and by multiplication on 
the right hand factor of $\Lambda^{t} \otimes S^{*}$; for $V \in \mathrm{Ob} \mathscr{V}^{f}$, this restricts to an isomorphism of $S^{*}(V)$-modules

$$
\bigoplus_{t \geqslant 0}\left(f_{t} S^{*} / f_{t-1} S^{*}\right)(V) \cong S^{*}(V),
$$

where $S^{*}(V)$ acts on the right hand side via $\Phi$.

3. For $V \in \mathrm{Ob}_{\mathscr{V}} f$, the inclusion $f_{t} S^{*}(V) \hookrightarrow S^{*}(V)$ is an isomorphism for $t \geqslant$ $\operatorname{dim} V$.

Proof. Straightforward; to prove that $S^{*}(V)$ is isomorphic to $\bigoplus_{t \geqslant 0}\left(f_{t} S^{*} / f_{t-1} S^{*}\right)(V)$ as $S^{*}(V)$-modules, it is sufficient to consider monomial bases.

For notational clarity, shifts in gradings are omitted from the following statement:

Proposition 6.3. For $i, t \in \mathbb{N}$, the Milnor derivation $Q_{i}: S^{*} \rightarrow S^{*}$ is a morphism of $\Phi S^{*}$-modules and restricts to $f_{t} S^{*} \stackrel{f_{t} Q_{i}}{\longrightarrow} f_{t-1} S^{*}$; the induced morphism on the filtration quotients

$$
\Lambda^{t} \otimes S^{*} \cong f_{t} S^{*} / f_{t-1} S^{*} \rightarrow f_{t-1} S^{*} / f_{t-2} S^{*} \cong \Lambda^{t-1} \otimes S^{*}
$$

is the Koszul differential $\tau_{i}$.

Proof. Straightforward.

Remark 6.4. The differentials $\tau_{0}$ and $\tau_{1}$ define a bicomplex structure on $\Lambda^{*} \otimes S^{*}$.

\subsection{Filtering the functors $K_{n}$}

This section establishes the filtered version of Proposition 4.9; the starting point is the functorial homology of the Koszul complexes, which is a standard result, related to Proposition 4.4:

Proposition 6.5. For $i \in \mathbb{N}$, the homology of $\left(\Lambda^{*} \otimes S^{*}, \tau_{i}\right)$ is $S^{*} /\left\langle x^{2^{i}}\right\rangle$, concentrated in homological degree 0.

The following are analogous to the functors $K_{n}$ introduced in Notation 4.6:

Notation 6.6. For an integer $a \geqslant 1$ and $b \in \mathbb{N}$, let $\mathfrak{K}_{a, b}$ denote the image of $\tau_{0}: \Lambda^{a} \otimes$ $S^{b} \rightarrow \Lambda^{a-1} \otimes S^{b+1}$; by convention, $\mathfrak{K}_{0, b}$ is $\mathbb{F}$ for $b=0$ and zero otherwise.

Lemma 6.7. For $0<n \in \mathbb{N}$, the filtration $f_{*} S^{*}$ induces a filtration of $K_{n}$ with associated graded $\operatorname{gr} K_{n} \cong \bigoplus_{a+2 b+1=n} \mathfrak{K}_{a, b}$.

Proof. For $n>0, K_{n}$ is the image of $Q_{0}: S^{n-1} \rightarrow S^{n}$. Passing to the associated graded, $Q_{0}$ induces

$$
\bigoplus \tau_{0}: \bigoplus_{a+2 b+1=n} \Lambda^{a} \otimes S^{b} \rightarrow \bigoplus_{a+2 b+1=n} \Lambda^{a-1} \otimes S^{b+1},
$$

by Proposition 6.3. Moreover, evaluated on $V \in \mathrm{Ob}^{f}$, as a morphism of vector spaces, $Q_{0}$ identifies with $\bigoplus \tau_{0}$, using the splitting of the filtration in $S^{*}(V)$-modules, given in Lemma 6.2. 
Lemma 6.8. The derivation $\tau_{1}$ induces a differential $\tau_{1}: \mathfrak{K}_{a+1, b-2} \rightarrow \mathfrak{K}_{a, b}$. For $a>0$ and $b \geqslant 0$, the short exact sequences from the Koszul complexes

$$
0 \rightarrow \mathfrak{K}_{a, b} \rightarrow \Lambda^{a-1} \otimes S^{b+1} \rightarrow \mathfrak{K}_{a-1, b+1} \rightarrow 0
$$

induce a short exact sequence of complexes

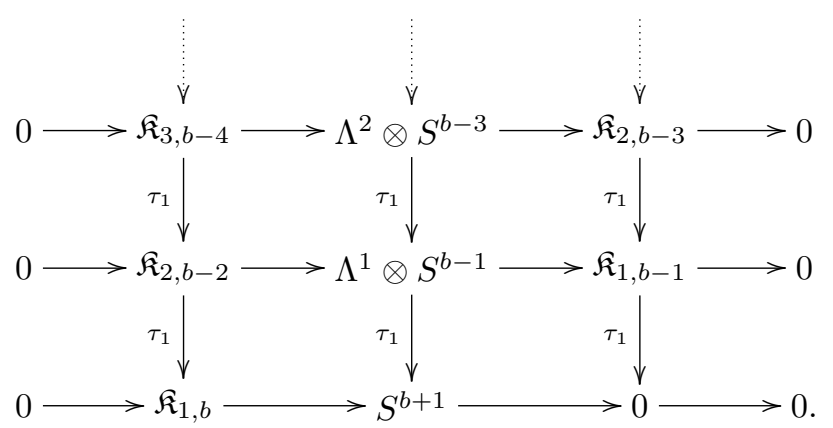

Proof. The horizontal $\tau_{0}$-Koszul complexes are acyclic.

Proposition 6.9. For $b \in \mathbb{N}$, the complex

$$
\cdots \rightarrow \mathfrak{K}_{n+1, b-2 n} \stackrel{\tau_{7}}{\rightarrow} \mathfrak{K}_{n, b-2 n+2} \rightarrow \cdots \rightarrow \mathfrak{K}_{1, b} \cong S^{b+1}
$$

has homology $p_{b+1} \bar{I}_{\mathbb{F}}$ concentrated in homological degree 0 .

Proof. The proof is by induction upon $b$, using the short exact sequence of complexes provided by Lemma 6.8. The initial case $b=0$ is by inspection; for the inductive step, use the fact that the $\tau_{1}$ Koszul complex

$$
\cdots \rightarrow \Lambda^{n} \otimes S^{b-2 n+1} \stackrel{\tau_{1}}{\rightarrow} \Lambda^{n-1} \otimes S^{b-2 n+3} \rightarrow \cdots \rightarrow S^{b+1}
$$

has homology $\Lambda^{b+1}$ concentrated in homological degree 0 , by Proposition 6.5 (with $i=$ 1 ). The proof is completed by the argument employed in the proof of Proposition 4.9.

\subsection{Filtering the functors $L_{n}$ and $\tilde{L}_{n}$}

Notation 6.10. For integers $a>0, b \geqslant 0$, let $\mathfrak{L}_{a, b}$ denote the cokernel of $\tau_{1}: \mathfrak{K}_{a+1, b-2} \rightarrow$ $\mathfrak{K}_{a, b}$.

Proposition 6.9 implies the following identification:

Lemma 6.11.

1. If $a>1, \mathfrak{L}_{a, b} \cong \operatorname{Ker}\left\{\mathfrak{K}_{a, b} \stackrel{\tau_{7}}{\rightarrow} \mathfrak{K}_{a-1, b+2}\right\}$.

2. $\mathfrak{L}_{1, b} \cong p_{b+1} \bar{I}_{\mathbb{F}}$.

Recall from Section 4.2 that $Q_{1}$ induces a morphism $Q_{1}: K_{n-3} \rightarrow K_{n}$ with image $L_{n}$ and kernel $\tilde{L}_{n-3}$. It follows that the cokernel of $Q_{1}$ occurs in an extension

$$
0 \rightarrow \tilde{L}_{n} / L_{n} \rightarrow \operatorname{Coker} Q_{1} \rightarrow L_{n+3} \rightarrow 0 .
$$


Lemma 6.12. For $n>0$ and $\operatorname{Coker} Q_{1}$ as above, the filtration $f_{\bullet} S^{*}$ induces a finite filtration of $\operatorname{Coker} Q_{1}$ with associated graded

$$
\operatorname{grCoker} Q_{1} \cong \bigoplus_{a+2 b+1=n, a \geqslant 1} \mathfrak{L}_{a, b},
$$

where, for $2(b+1)=n$, the sub-object $\mathfrak{L}_{1, b}$ is isomorphic to $\tilde{L}_{2(b+1)} / L_{2(b+1)} \cong p_{b+1} \bar{I}_{\mathbb{F}}$ and there is an induced isomorphism $\operatorname{gr} L_{n+3} \cong \bigoplus_{a+2 b+1=n, a \geqslant 2} \mathfrak{L}_{a, b}$.

Proof. The result follows as for the proof of Lemma 6.7.

Remark 6.13. For the calculations of local cohomology, it is important that the isomorphisms of Lemma 6.7 and of Lemma 6.12 upon evaluation on $V \in \mathrm{Ob}_{\mathscr{V}}{ }^{f}$ correspond to isomorphisms in the category of $S^{*}(V)$-modules (as in Lemma 6.2).

\section{Local duality}

An equivariant version of local duality (with respect to the action of the general linear groups $\operatorname{Aut}(V)$ ) is given as it arises in the current context, refining results of [BG03, Section 4.7].

\subsection{Categories of $S^{*}$-modules}

Throughout this section, the prime $p$ is arbitrary.

\section{Definition 7.1.}

1. Let $S^{*}-\bmod _{\mathscr{F}}$ denote the category of graded right $S^{*}$-modules in $\mathscr{F}$ and $S^{*}$ module morphisms.

2. For $V \in \mathrm{Ob}^{\mathscr{V}} f$, let $S^{*}(V)-\bmod _{\mathrm{Aut}(V)}$ denote the category of graded right $S^{*}(V)$-modules in $\operatorname{Aut}(V)$-modules and $S^{*}(V)$-module morphisms.

Lemma 7.2. For $V \in \mathrm{Ob} \mathscr{V}^{f}$, the categories $S^{*}-\bmod \mathscr{F}$ and $S^{*}(V)-\bmod _{\mathrm{Aut}(V)}$ are abelian. Moreover:

1. the forgetful functor $S^{*}-\bmod _{\mathscr{F}} \rightarrow \mathscr{F}$ is exact and admits an exact left adjoint $-\otimes S^{*}: \mathscr{F} \rightarrow S^{*}-\bmod _{\mathscr{F}} ;$

2. the forgetful functor $S^{*}(V)-\bmod _{\mathrm{Aut}(V)} \rightarrow \operatorname{Aut}(V)-\bmod$ is exact and admits an exact left adjoint $-\otimes S^{*}(V): \operatorname{Aut}(V)-\bmod \rightarrow S^{*}(V)-\bmod _{\mathrm{Aut}(V)}$;

3. evaluation at $V, \mathscr{F} \rightarrow \operatorname{Aut}(V)-\mathbf{m o d}$, induces an exact functor $S^{*}-\bmod \mathbf{F}_{\mathscr{F}} \rightarrow$ $S^{*}(V)-\bmod _{\mathrm{Aut}(V)}$.

Proof. Clear.

Definition 7.3. For $N \in \mathrm{Ob} S^{*}-\bmod \mathscr{F}_{\mathscr{F}}$, let $\operatorname{Hom}_{S^{*}}^{V}(-, N)$ be the functor

$$
\left(S^{*}-\bmod _{\mathscr{F}}\right)^{\mathrm{op}} \rightarrow S^{*}(V)-\bmod _{\mathrm{Aut}(V)}
$$

defined by

$$
\operatorname{Hom}_{S^{*}}^{V}(M, N):=\operatorname{Hom}_{S^{*}(V)}(M(V), N(V)),
$$

where the right-hand side is equipped with the usual grading and $\operatorname{Aut}(V)$ acts via conjugation. 
Lemma 7.4. For $F \in \mathrm{Ob} \mathscr{F}$ and $V \in \mathrm{Ob} \mathscr{V} f$, there is a natural isomorphism

$$
\operatorname{Hom}_{S^{*}}^{V}\left(F \otimes S^{*}, S^{*}\right) \cong F(V)^{\sharp} \otimes S^{*}(V)
$$

in $\operatorname{Aut}(V)-$ mod, where $F(V)^{\sharp}$ is equipped with the contragredient action.

Proof. Straightforward.

In terms of the above identification, the following is straightforward:

Lemma 7.5. Let $F, G \in \mathrm{Ob} \mathscr{F}, V \in \mathrm{Ob}_{\mathscr{V}}{ }^{f}$, and $\alpha: F \otimes S^{*} \rightarrow G \otimes S^{*}$ be a morphism of $S^{*}-\bmod _{\mathscr{F}}$, induced by $\tilde{\alpha}: F \rightarrow G \otimes S^{*}$ in $\mathscr{F}$.

Then $\operatorname{Hom}_{S^{*}}^{V}\left(\alpha, S^{*}\right)$ identifies with the morphism $G(V)^{\sharp} \otimes S^{*}(V) \rightarrow$ $F(V)^{\sharp} \otimes S^{*}(V)$ of $S^{*}(V)-\bmod _{\mathrm{Aut}(V)}$ induced by $\gamma: G(V)^{\sharp} \rightarrow F(V)^{\sharp} \otimes S^{*}(V)$ in $\operatorname{Aut}(V)-$ mod, which is adjoint to $\left(\tilde{\alpha}_{V}\right)^{\sharp}: G(V)^{\sharp} \otimes S^{*}(V)^{\sharp} \rightarrow F(V)^{\sharp}$ (in $\operatorname{Aut}(V)-$ $\bmod )$.

\subsection{The dualizing functor}

Self-duality of the exterior power functors gives:

Lemma 7.6. For $n \in \mathbb{N}$, there is a natural isomorphism of contravariant functors of $V: \Lambda^{n}\left(V^{\sharp}\right) \cong \Lambda^{n}(V)^{\sharp}$.

This is combined with the following duality result, when restricting to the consideration of the $\operatorname{Aut}(V)$-action (henceforth $V_{r}$ denotes an object of $\mathrm{Ob}^{f}{ }^{f}$ of rank $r)$.

Lemma 7.7. Let $0 \leqslant j \leqslant r$ be integers. The composite

$$
\Lambda^{r}\left(V_{r}\right) \otimes \Lambda^{j}\left(V_{r}\right) \stackrel{\Delta \otimes 1}{\rightarrow} \Lambda^{r-j}\left(V_{r}\right) \otimes \Lambda^{j}\left(V_{r}\right) \otimes \Lambda^{j}\left(V_{r}\right)^{\sharp} \rightarrow \Lambda^{r-j}\left(V_{r}\right),
$$

where the second morphism is induced by evaluation $\Lambda^{j}\left(V_{r}\right) \otimes \Lambda^{j}\left(V_{r}\right)^{\sharp} \rightarrow \mathbb{F}$, induces an isomorphism $\Lambda^{r}\left(V_{r}\right) \otimes \Lambda^{j}\left(V_{r}\right)^{\sharp} \cong \Lambda^{r-j}\left(V_{r}\right)$ in $\operatorname{Aut}\left(V_{r}\right)-\bmod$, where $\Lambda^{j}\left(V_{r}\right)^{\sharp}$ is equipped with the contragredient $\operatorname{Aut}\left(V_{r}\right)$-module structure.

Proof. The result follows from the fact that the product $\Lambda^{r-j}\left(V_{r}\right) \otimes \Lambda^{j}\left(V_{r}\right) \rightarrow$ $\Lambda^{r}\left(V_{r}\right) \cong \mathbb{F}$ defines a perfect pairing and the equivariance of the evaluation map.

Lemma 7.8. Let $1 \leqslant j \leqslant r$ be integers, and write $\mu: \Lambda^{j-1}\left(V_{r}\right)^{\sharp} \otimes \Lambda^{1}\left(V_{r}\right)^{\sharp} \rightarrow \Lambda^{j}\left(V_{r}\right)^{\sharp}$ for the product morphism (dual to the the coproduct $\Lambda^{j}\left(V_{r}\right) \rightarrow \Lambda^{j-1}\left(V_{r}\right) \otimes \Lambda^{1}\left(V_{r}\right)$ ).

Then, under the isomorphism of Lemma 7.7, $\Lambda^{r}\left(V_{r}\right) \otimes \mu$ is Aut $\left(V_{r}\right)$-equivariantly isomorphic to the morphism

$$
\Lambda^{r-j+1}\left(V_{r}\right) \otimes \Lambda^{1}\left(V_{r}\right)^{\sharp} \rightarrow \Lambda^{r-j}\left(V_{r}\right)
$$

which is adjoint to the coproduct $\Lambda^{r-j+1}\left(V_{r}\right) \rightarrow \Lambda^{r-j}\left(V_{r}\right) \otimes \Lambda^{1}\left(V_{r}\right)$.

Proof. A consequence of the coassociativity of the comultiplication on the exterior power functors and the fact that the multiplication $\mu$ is dual to the coproduct $\Lambda^{j} \rightarrow$ $\Lambda^{j-1} \otimes \Lambda^{1}$.

Notation 7.9. For $i \in \mathbb{N}$, 
1. let $\tau_{i}: \Lambda^{1} \rightarrow S^{p^{i}}$ denote both the composite of the isomorphism $\Lambda^{1} \cong S^{1}$ with the iterated Frobenius $S^{1} \hookrightarrow S^{p^{i}}$ and the induced Koszul differential $\tau_{i}: \Lambda^{j} \otimes$ $S^{*} \rightarrow \Lambda^{j-1} \otimes S^{*+p^{i}}$ in the category $S^{*}-\bmod _{\mathscr{F}}$,

2. let $\mathbf{K} \mathbf{z}_{i}$ denote the Koszul complex in $S^{*}-\bmod \mathscr{F}$ :

$$
\cdots \rightarrow \Lambda^{j} \otimes S^{*-j p^{i}} \stackrel{\tau_{i}}{\rightarrow} \Lambda^{j-1} \otimes S^{*-(j-1) p^{i}} \rightarrow \cdots \rightarrow S^{*} \rightarrow 0,
$$

where $\tau_{i}$ is as above, and

3. let $\mathbf{K} \mathbf{z}_{i}\left(V_{r}\right)$ denote the evaluated Koszul complex in $S^{*}\left(V_{r}\right)-\bmod _{\mathrm{Aut}\left(V_{r}\right)}$.

Proposition 7.10. For integers $1 \leqslant j \leqslant r$ and $i \in \mathbb{N}$, under the isomorphism of Lemma 7.4, the morphism

$$
\operatorname{Hom}_{S^{*}}^{V_{r}}\left(\tau_{i}, S^{*}\right): \operatorname{Hom}_{S^{*}}^{V_{r}}\left(\Lambda^{j-1} \otimes S^{*}, S^{*}\right) \rightarrow \operatorname{Hom}_{S^{*}}^{V_{r}}\left(\Lambda^{j} \otimes S^{*}, S^{*}\right)
$$

is induced by the morphism $\gamma: \Lambda^{j-1}\left(V_{r}\right)^{\sharp} \rightarrow \Lambda^{j}\left(V_{r}\right)^{\sharp} \otimes S^{p^{i}}\left(V_{r}\right)$ such that, under the isomorphism of Lemma 7.7, $\Lambda^{r}\left(V_{r}\right) \otimes \gamma$ is $\operatorname{Aut}\left(V_{r}\right)$-equivariantly isomorphic to the Koszul differential $\tau_{i}\left(V_{r}\right): \Lambda^{r-j+1}\left(V_{r}\right) \rightarrow \Lambda^{r-j}\left(V_{r}\right) \otimes S^{p^{i}}\left(V_{r}\right)$.

Proof. Combine Lemma 7.5 with Lemma 7.8.

Corollary 7.11. For $i \in \mathbb{N}$, there is a natural isomorphism of complexes

$$
\operatorname{Hom}_{S^{*}}^{V_{r}}\left(\mathbf{K z}_{i}, \Lambda^{r} \otimes S^{*}\right) \cong \mathbf{K z}_{i}\left(V_{r}\right)
$$

in $S^{*}\left(V_{r}\right)-\bmod _{\mathrm{Aut}\left(V_{r}\right)}$.

Proof. After addition of the "twisting functor" $\Lambda^{r}$, the result is an immediate consequence of Proposition 7.10.

Remark 7.12. Taking $i=0$, so that $\mathbf{K z}_{0}$ is the usual Koszul complex, this shows that $\Lambda^{r} \otimes S^{*}$ is the dualizing object, corresponding to the fact that $S^{*}$ is graded Gorenstein (cf. [BH93, Section 3.7]).

\subsection{Local cohomology in $S^{*}\left(V_{r}\right)-\bmod _{\mathrm{Aut}\left(V_{r}\right)}$}

In this section, local cohomology is considered with respect to the augmentation ideal $I$ of $S^{*}\left(V_{r}\right)$, so that $I \cong \bigoplus_{t>0} S^{t}\left(V_{r}\right)$. The results of the previous section show that the local duality isomorphism (cf. [BH93]) should be interpreted as stating that the local cohomology of $F\left(V_{r}\right) \otimes S^{*}\left(V_{r}\right)$, for $F \in \mathrm{Ob} \mathscr{F}$, is concentrated in cohomological degree $r$, where it is isomorphic to $\operatorname{Hom}_{\mathscr{V} f}\left(\operatorname{Hom}_{S^{*}}^{V_{r}}\left(F \otimes S^{*}, \Lambda^{r} \otimes S^{*}\right), \mathbb{F}\right)$.

Proposition 7.13. (cf. [BG03, Lemma 4.7.1]) Let $G \in \mathrm{Ob} S^{*}-\bmod _{\mathscr{F}}$ such that there exists a complex

$$
0 \rightarrow F_{r} \otimes S^{*} \rightarrow F_{r-1} \otimes S^{*} \rightarrow \cdots \rightarrow F_{0} \otimes S^{*} \rightarrow G \rightarrow 0
$$

that induces an exact sequence in $S^{*}\left(V_{r}\right)-\bmod _{\mathrm{Aut}\left(V_{r}\right)}$, by evaluation on $V_{r}$. Then the local cohomology of $G\left(V_{r}\right) \in \mathrm{Ob} S^{*}\left(V_{r}\right)-\bmod _{\mathrm{Aut}\left(V_{r}\right)}$ is

$$
H_{I}^{i}\left(G\left(V_{r}\right)\right) \cong H_{r-i}\left(\operatorname{Hom}_{V^{f}}\left(\operatorname{Hom}_{S^{*}}^{V_{r}}\left(F_{i} \otimes S^{*}, \Lambda^{r} \otimes S^{*}\right), \mathbb{F}\right)\right),
$$

up to shift in grading. 


\section{The local cohomology spectral sequence}

The local cohomology theorem for $k u$ implies that there is a spectral sequence

$$
E^{2}:=H_{I}^{*, *}\left(k u^{*}\left(B V_{+}\right)\right) \Rightarrow k u_{*}\left(B V_{+}\right),
$$

where the $E^{2}$-term is the local cohomology with respect to the augmentation ideal (see [BG03] for generalities on the spectral sequence, for arbitrary finite groups). Here $V=V_{r}$; the subscript is omitted for typographical reasons.

The aim of this section is to indicate how the spectral sequence can be understood conceptually. It can be made $\operatorname{Aut}(V)$-equivariant but is not functorial with respect to arbitrary vector space morphisms (local cohomology is sensitive to Krull dimension); however, the behaviour of the spectral sequence is largely determined by functorial structure. There are two key ingredients: the functorial description of local duality and of local cohomology given in Section 7 and the relationship between the local

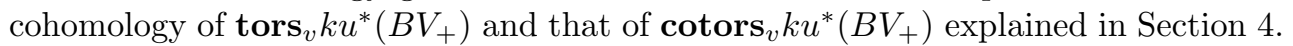

These results were inspired greatly by the work of Bruner and Greenlees [BG03], who discovered the fundamental behaviour exhibited by the local cohomology of $\operatorname{tors}_{v} k u^{*}\left(B V_{+}\right)$.

Remark 8.1. Throughout, the grading shifts resulting from working with graded modules are suppressed. The gradings are not essential for the presentation of the arguments; the reader is, however, urged to calculate them.

\subsection{The case of integral cohomology}

The local cohomology spectral sequence for $H \mathbb{Z}_{*}\left(B V_{+}\right)$already illustrates some of the salient features. It can be used in the analysis of the local cohomology spectral sequence for $k u_{*}\left(B V_{+}\right)$via the morphism induced by $k u \rightarrow H \mathbb{Z}$.

Let $V \in \mathrm{Ob} \mathscr{V}^{f}$ have rank $r$, and consider the short exact sequence

$$
0 \rightarrow H \mathbb{Z}^{*}(B V) \rightarrow H \mathbb{Z}^{*}\left(B V_{+}\right) \rightarrow \mathbb{Z} \rightarrow 0
$$

in the category of $H \mathbb{Z}^{*}\left(B V_{+}\right)$-modules, so that $H \mathbb{Z}^{*}(B V)$ corresponds to the augmentation ideal $I$. There is an induced exact sequence of local cohomology groups

$$
\begin{aligned}
0 \rightarrow H_{I}^{0}\left(H \mathbb{Z}^{*}(B V)\right) \rightarrow H_{I}^{0}\left(H \mathbb{Z}^{*}\left(B V_{+}\right)\right) & \rightarrow \\
\rightarrow \mathbb{Z} & \rightarrow H_{I}^{1}\left(H \mathbb{Z}^{*}(B V)\right) \rightarrow H_{I}^{1}\left(H \mathbb{Z}^{*}\left(B V_{+}\right)\right) \rightarrow 0
\end{aligned}
$$

and, for $j>1$, a natural isomorphism $H_{I}^{j}\left(H \mathbb{Z}^{*}\left(B V_{+}\right)\right) \cong H_{I}^{j}\left(H \mathbb{Z}^{*}(B V)\right)$.

Hence, up to calculating the connecting morphism $\mathbb{Z} \rightarrow H_{I}^{1}\left(H \mathbb{Z}^{*}(B V)\right)$, the local cohomology of $H \mathbb{Z}^{*}\left(B V_{+}\right)$is determined by that of $H \mathbb{Z}^{*}(B V)$. Moreover, since $H \mathbb{Z}^{*}(B V)$ is annihilated by 2 , so is $H_{I}^{1}\left(H \mathbb{Z}^{*}(B V)\right)$; hence it suffices to consider behaviour after reducing mod 2 .

Notation 8.2. For $a \in \mathbb{N}$, let $\sigma \geqslant_{a} \mathbf{K z}_{0}$ denote the brutal truncation of the Koszul complex to the right

$$
\cdots \rightarrow \Lambda^{a+1} \otimes S^{*-1} \stackrel{\tau_{0}}{\rightarrow} \Lambda^{a} \otimes S^{*}
$$

and $\sigma_{\leqslant a} \mathbf{K z}_{0}$ the brutal truncation to the left

$$
\Lambda^{a} \otimes S^{*} \stackrel{\tau_{0}}{\rightarrow} \Lambda^{a-1} \otimes S^{*+1} \stackrel{\tau_{0}}{\rightarrow} \cdots \rightarrow S^{*+a} .
$$


Lemma 8.3. For $a \in \mathbb{N}$,

1. $\sigma_{\geqslant a} \mathbf{K z}_{0}$ is an $S^{*}$-free resolution of $\mathfrak{K}_{a, *}$, and

2. for $a \geqslant 1$, the complex $\sigma_{\leqslant a} \mathbf{K z}_{0}$ has homology $\mathbb{F}$ in homological degree 0 and $\mathfrak{K}_{a+1, *}$ in homological degree $a$.

Moreover, there are morphisms of complexes

$$
\begin{aligned}
& \mathbf{K} \mathbf{z}_{0} \rightarrow \sigma_{\geqslant 1} \mathbf{K} \mathbf{z}_{0} \rightarrow \sigma_{\geqslant 2} \mathbf{K} \mathbf{z}_{0} \rightarrow \cdots \\
& \sigma_{\leqslant 0} \mathbf{K} \mathbf{z}_{0} \cong S^{*} \hookrightarrow \sigma_{\leqslant 1} \mathbf{K} \mathbf{z}_{0} \hookrightarrow \sigma_{\leqslant 2} \mathbf{K} \mathbf{z}_{0} \hookrightarrow \cdots \subset \mathbf{K} \mathbf{z}_{0} .
\end{aligned}
$$

Proof. Clear.

Corollary 7.11 gives the following:

Proposition 8.4. For integers $0 \leqslant a \leqslant b \leqslant r$, there is a natural isomorphism of complexes

$$
\operatorname{Hom}_{S^{*}}^{V_{r}}\left(\sigma_{\geqslant a} \mathbf{K} \mathbf{z}_{0}, S^{*} \otimes \Lambda^{r}\right) \cong \sigma_{\leqslant r-a} \mathbf{K} \mathbf{z}_{0}\left(V_{r}\right)
$$

in $S^{*}\left(V_{r}\right)-\bmod _{\mathrm{Aut}\left(V_{r}\right)}$, and, with respect to these isomorphisms, the surjection $\sigma_{\geqslant a} \mathbf{K} \mathbf{z}_{0} \rightarrow \sigma_{\geqslant b} \mathbf{K} \mathbf{z}_{0}$ induces the inclusion $\sigma_{\leqslant r-b} \mathbf{K} \mathbf{z}_{0}\left(V_{r}\right) \hookrightarrow \sigma_{\leqslant r-a} \mathbf{K z}_{0}\left(V_{r}\right)$.

In particular, the surjection $\mathbf{K} \mathbf{z}_{0} \rightarrow \sigma_{\geqslant b} \mathbf{K} \mathbf{z}_{0}$ induces $\sigma_{\leqslant r-b} \mathbf{K} \mathbf{z}_{0}\left(V_{r}\right) \hookrightarrow \mathbf{K} \mathbf{z}_{0}\left(V_{r}\right)$, which gives an isomorphism in degree 0 homology if $b<r$.

Remark 8.5. It is useful to think of the surjection $\sigma_{\geqslant a} \mathbf{K z}_{0} \rightarrow \sigma_{\geqslant b} \mathbf{K z}_{0}$ as a morphism $\mathfrak{K}_{a, *}[a] \rightarrow \mathfrak{K}_{b, *}[b]$ in an appropriate derived category, where $[a]$, $[b]$ correspond to the shift in homological degree. In particular, for $a=0$, this corresponds to $\mathbb{F} \rightarrow \mathfrak{K}_{b, *}[b]$.

The morphism $\sigma_{\geqslant a} \mathbf{K z}_{0} \rightarrow \sigma_{\geqslant b} \mathbf{K z}_{0}$ induces a morphism between local cohomology groups, via the identification of local cohomology given in Proposition 7.13 and Proposition 8.4.

Using the above observations, one deduces the following lemma:

Lemma 8.6. For $V \in \mathrm{Ob} \mathscr{V}^{f}$ of rank $r>1$, the connecting morphism $H_{I}^{0}(\mathbb{Z} / 2) \cong$ $\mathbb{Z} / 2 \rightarrow H_{I}^{1}\left(H \mathbb{Z}^{*}(B V)\right)$ induced by the short exact sequence of $H \mathbb{Z}^{*}\left(B V_{+}\right)$-modules

$$
0 \rightarrow H \mathbb{Z}^{*}(B V) \rightarrow H \mathbb{Z}^{*}\left(B V_{+}\right) / 2 \rightarrow \mathbb{Z} / 2 \rightarrow 0
$$

is non-trivial.

Remark 8.7. The connecting morphism in the long exact sequence (2) for local cohomology is therefore non-trivial. For a conceptual presentation of the results, it is useful to define an associated $E^{1}$-page, so that this connecting morphism appears as $d^{1}$, as in [BG03].

The local cohomology $(r>1)$ is as follows, using Lemma 8.3:

$$
H_{I}^{j}\left(H \mathbb{Z}^{*}\left(B V_{+}\right)\right) \cong \begin{cases}\mathbb{Z} & j=0 \\ 0 & j=1 \\ \mathbb{F} & 2 \leqslant j \leqslant r-1 \\ H_{I}^{r}\left(H \mathbb{Z}^{*}(B V)\right) & j=r .\end{cases}
$$

Moreover, $H_{I}^{r}\left(H \mathbb{Z}^{*}(B V)\right)$ has a finite filtration such that

$$
\operatorname{gr} H_{I}^{r}\left(H \mathbb{Z}^{*}(B V)\right) \cong \mathbb{F} \oplus \operatorname{gr} H \mathbb{Z}_{*}(B V),
$$

up to shift in degree, where the filtration of $H \mathbb{Z}_{*}(B V)$ is induced by $f_{t} S^{*}$. 
The analysis of the local cohomology spectral sequence is straightforward; the local cohomology corresponds to the $E^{2}$-page of the spectral sequence. The permanent cycles in the zero column are given by the subgroup $2^{r-1} \mathbb{Z}$; the differentials $d^{i}$, for $2 \leqslant i \leqslant r$ are all non-trivial, starting from the zero column, and serve to eliminate the extraneous factors of $\mathbb{F}$ that occur above.

Remark 8.8. Heuristically, the differential $d^{i}$ is induced by the surjection of complexes $\mathbf{K} \mathbf{z}_{0} \rightarrow \sigma_{\geqslant i} \mathbf{K} \mathbf{z}_{0}$, for $i \geqslant 1$.

\subsection{Bicomplexes of $S^{*}$-modules}

The purpose of this section is to explain the calculation of the local cohomology of $\operatorname{tors}_{v} k u^{*}\left(B V_{+}\right)$; a fundamental point is that the method also calculates the local

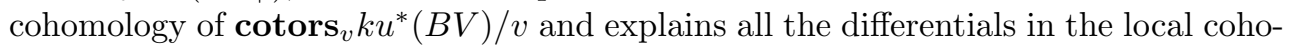
mology spectral sequence. This relies on the following result, in which grading shifts have been suppressed and, for variance reasons, the cohomology of $V^{\sharp}$ is considered.

Proposition 8.9. (Cf. [BG03, Section 4.6].) For $V \in \mathrm{Ob}^{f} f$ of $\operatorname{rank} r$, $\operatorname{tors}_{v} k u^{*}\left(B V_{+}^{\sharp}\right)$ admits a finite natural filtration with associated graded

$$
\operatorname{grtors}_{v} k u^{*}\left(B V_{+}^{\sharp}\right) \cong \bigoplus_{i=2}^{r} \mathfrak{L}_{i, *}(V)
$$

in $S^{*}(V)-\bmod _{\mathrm{Aut}(V)}$. Moreover, as a module over $S^{*}(V)$,

$$
\operatorname{tors}_{v} k u^{*}\left(B V_{+}^{\sharp}\right) \cong \bigoplus_{i=2}^{r} \mathfrak{L}_{i, *}(V) .
$$

Proof. The result follows from Lemma 6.12 and Theorem 5.13.

For the consideration of local duality, consider the $S^{*}$-action on the bicomplexes introduced in Section 6.1 and the following truncations, which are analogues of the truncated Koszul complexes of Notation 8.2.

Definition 8.10. (cf. [BG03, Section 4.6]) For $i \in \mathbb{N}$,

1. let $\mathbf{B}(i)$ be the bicomplex in $S^{*}-\bmod \mathscr{F}$,

$$
\mathbf{B}(i)_{s, t}:= \begin{cases}0 & t<i \text { or } s<0 \\ \Lambda^{s+t} \otimes S^{*} & t \geqslant i\end{cases}
$$

considered as a quotient bicomplex, where the term of lowest total degree is $\Lambda^{i} \otimes S^{*}$, in bidegree $(0, i)$, and

2. let $\mathbf{D}(i)$ be the bicomplex in $S^{*}-\bmod _{\mathscr{F}}$,

$$
\mathbf{D}(i)_{s, t}:= \begin{cases}0 & t>i \text { or } s>0 \\ \Lambda^{s+t} \otimes S^{*} & t \leqslant i\end{cases}
$$

considered as a sub-bicomplex, where the term of greatest total degree is $\Lambda^{i} \otimes$ $S^{*}$, in bidegree $(0, i)$.

Remark 8.11.

1. When evaluated on $V_{r}$, the only non-trivial terms are those with $s+t \leqslant r$ and $t \geqslant i$. In particular, $\mathbf{B}(i)\left(V_{r}\right)$ is trivial if $i>r$. 
2. The grading on $\mathbf{B}(i)$ used in [BG03, Chapter 4] (respectively on $\mathbf{D}(i)$ ) can be recovered by considering the grading of the "generators" in the lowest (resp. greatest) total degree, since the morphism $\tau_{0}$ raises the degree by 2 and $\tau_{1}$ raises the degree by 4 (the grading is calculated relative to the $S^{*}$-grading, so that the usual gradings of the odd degree generators do not contribute).

3. The homology of the bicomplexes is calculated pointwise, by first evaluating on $V \in \mathrm{Ob}_{\mathscr{V}}$; for any $i$ and $V$, the bicomplex $\mathbf{B}(i)(V)$ has only finitely many non-zero terms.

The following is clear from the definitions:

Lemma 8.12.

1. There are surjections of bicomplexes in $S^{*}-\bmod _{\mathscr{F}}$

$$
\mathbf{B}(0) \rightarrow \mathbf{B}(1) \rightarrow \cdots \rightarrow \mathbf{B}(i) \rightarrow \mathbf{B}(i+1) \rightarrow \cdots,
$$

and the kernel of $\mathbf{B}(i) \rightarrow \mathbf{B}(i+1)$ is the truncated Koszul complex $\sigma_{\geqslant i} \mathbf{K} \mathbf{z}_{0}$, concentrated in $t$-degree $i$.

2. There are inclusions of bicomplexes

$$
\mathbf{D}(0)=S^{*} \rightarrow \mathbf{D}(1) \hookrightarrow \mathbf{D}(2) \hookrightarrow \cdots \hookrightarrow \mathbf{D}(j-1) \hookrightarrow \mathbf{D}(j) \hookrightarrow \cdots,
$$

and the cokernel of $\mathbf{D}(j-1) \hookrightarrow \mathbf{D}(j)$ is the truncated Koszul complex $\sigma_{\leqslant j} \mathbf{K z}_{0}$ shifted so that the term of maximal total degree is in bidegree $(0, j)$.

The following result follows from the definition of the objects $\mathfrak{K}_{a, b}$ and $\mathfrak{L}_{a, b}$ given in Section 4.

Lemma 8.13. For $0<a \in \mathbb{Z}, \mathfrak{K}_{a, *}$ and $\mathfrak{L}_{a, *}$ are objects of $S^{*}-\bmod \mathscr{F}_{\mathscr{F}}$ such that the surjections $\Lambda^{a} \otimes S^{*} \rightarrow \mathfrak{K}_{a, *} \rightarrow \mathfrak{L}_{a, *}$ are morphisms of $S^{*}-\bmod \boldsymbol{F}_{\mathscr{F}}$.

Remark 8.14. For $a \geqslant 2, \mathfrak{L}_{a, b}$ is a quotient of $\Lambda^{a} \otimes S^{b}$ and a sub-object of $\Lambda^{a-2} \otimes$ $S^{b+3}$.

In the following, recall the indexing convention of $\mathbf{B}(i)$, which means that the term of lowest total degree is in $(s, t)$-degree $(0, i)$, and hence has total degree $i$.

Proposition 8.15. For $0<i \in \mathbb{N}$, the homology of $\operatorname{Tot}(\mathbf{B}(i))$ is concentrated in degree $i$ and $H_{i}(\operatorname{Tot}(\mathbf{B}(i))) \cong \mathfrak{L}_{i, *}$.

Proof. The result follows by filtering the bicomplex $\mathbf{B}(i)$ using Lemma 8.12 and Proposition 6.9 (cf. [BG03, Proposition 4.6.3]).

Proposition 8.16. For $i \in \mathbb{N}$, the homology of $\operatorname{Tot}(\mathbf{D}(i))$ is as follows:

For $i=0$ :

$$
H_{m}(\operatorname{Tot}(\mathbf{D}(0))) \cong \begin{cases}S^{*} & m=0 \\ 0 & \text { otherwise. }\end{cases}
$$

For $i>0$ :

$$
H_{m}(\operatorname{Tot}(\mathbf{D}(i))) \cong \begin{cases}\mathbb{F}^{\oplus i+1} \oplus \bigoplus_{d \geqslant 1} p_{d} \bar{I}_{\mathbb{F}} & m=0 \\ \mathfrak{L}_{i+2, *} & m=i \\ 0 & \text { otherwise. }\end{cases}
$$

Moreover, for $i>1$, the short exact sequence of bicomplexes $0 \rightarrow \mathbf{D}(i-1) \rightarrow$ 
$\mathbf{D}(i) \rightarrow \sigma_{\leqslant i} \mathbf{K z}_{0} \rightarrow 0$ given by Lemma 8.12, induces the short exact sequences

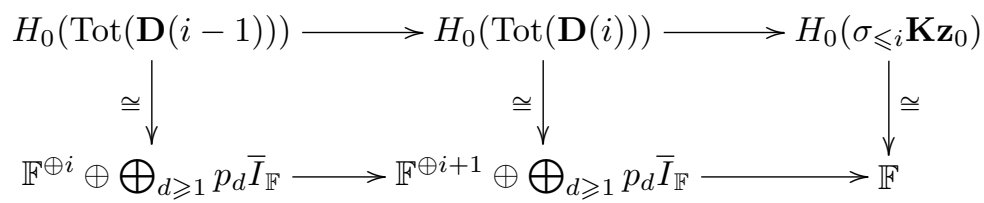

and

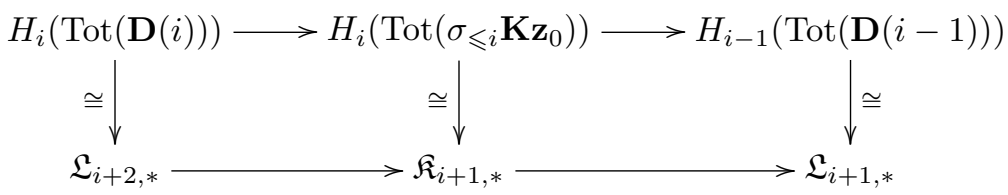

in homology.

Proof. The calculation of the homology follows from Proposition 6.9, together with the fact that each row of $\mathbf{D}(i)$, considered as a truncated Koszul complex, contributes a factor $\mathbb{F}$ in homological degree 0 .

For $i>1$, the given short exact sequences follow immediately; the exactness of the second sequence is again a consequence of Proposition 6.9.

Remark 8.17 .

1. The factors $\mathbb{F}$ (resp. the different factors $p_{d} \bar{I}_{\mathbb{F}}$ ) lie in distinct gradings.

2. The degree $r-1$ homology of $\mathbf{D}(r-1)$ is the functor $\mathfrak{L}_{r+1, *}$, which is a quotient of $\Lambda^{r+1} \otimes S^{*}$. In particular, when evaluated on the rank $r$ space $V$, this is trivial. Thus the homology of $\mathbf{D}(r-1)$ evaluated on $V$ is concentrated in degree 0 .

The following result is proved by using the identification of local cohomology given by Proposition 7.13. Here $D$ denotes the graded duality functor and the identification $D p_{d} \bar{I}_{\mathbb{F}} \cong q_{d} \bar{P}_{\mathbb{F}}$ is used to give the duality statement $D \mathfrak{L}_{1, *} \cong \bigoplus_{d>0} q_{d} \bar{P}_{\mathbb{F}} ;$ all functors should be understood as being evaluated on $V$.

Corollary 8.18. (cf. [BG03, Theorem 4.7.3]) For $V \in \mathrm{Ob}_{\mathscr{V}} f$ of rank $r>1$, the local cohomology of $\mathfrak{L}_{i, *}$ :

1. for $i=1$ is concentrated in cohomological degree 1 and $H_{I}^{1}\left(\mathfrak{L}_{1, *}\right) \cong \mathbb{F}^{\oplus r} \oplus D \mathfrak{L}_{1, *}$;

2. for $2 \leqslant i \leqslant r-1$,

$$
H_{I}^{m}\left(\mathfrak{L}_{i, *}\right) \cong \begin{cases}\mathbb{F}^{\oplus r-i+1} \oplus D \mathfrak{L}_{1, *} & m=i \\ D \mathfrak{L}_{r-i+2, *} & m=r \\ 0 & \text { otherwise; }\end{cases}
$$

3. for $i=r$ is concentrated in cohomological degree $r$ and $H_{I}^{r}\left(\mathfrak{L}_{r, *}\right) \cong D S^{*}$.

Moreover, the surjection of complexes $\mathbf{B}(1) \rightarrow \mathbf{B}(i)$, for $1<i<r$, induces a surjection: $H_{I}^{1}\left(\mathfrak{L}_{1, *}\right) \rightarrow H_{I}^{i}\left(\mathfrak{L}_{i, *}\right)$ with kernel $\mathbb{F}^{\oplus i-1}$.

\section{Remark 8.19.}

1. The restriction $r>1$ on the rank is imposed so as to give a unified statement. 
2. The grading is again suppressed; the reader is encouraged to calculate the appropriate gradings and to verify that the above yields the Hilbert series specified in [BG03, Section 4.7].

3. A notational sleight of hand has been used; the local cohomology introduces a change of variance with respect to $V ; \mathfrak{L}_{i, *}$ on the left-hand side should be considered as being contravariant in $V$, by precomposition with the duality functor $\sharp$.

\subsection{The local cohomology of $\operatorname{cotors}_{v} k u^{*}\left(B V_{+}\right)$}

Throughout this section, $V \in \mathrm{Ob}^{f}$ has rank $r>1$. To simplify notation, $Q$ will be written for the (contravariant) functor $V \mapsto \operatorname{cotors}_{v} k u^{*}\left(B V_{+}\right)$. Multiplication by $v$ induces a short exact sequence

$$
0 \rightarrow Q \stackrel{v}{\rightarrow} Q \rightarrow Q / v \rightarrow 0
$$

(omitting the suspension associated with the grading). Moreover, the augmentation $Q \rightarrow \mathbb{Z}[v]$ induces a short exact sequence

$$
0 \rightarrow \bar{Q} / v \rightarrow Q / v \rightarrow \mathbb{Z} \rightarrow 0 .
$$

Theorem 5.13 yields the natural isomorphism $\bar{Q} / v \cong \mathfrak{L}_{1, *}\left(V^{\sharp}\right)$; hence, by using the change of rings isomorphism associated to $k u^{*}\left(B V_{+}\right) \rightarrow H \mathbb{Z}^{*}\left(B V_{+}\right)$, Corollary 8.18 gives the local cohomology of $\bar{Q} / v$. In particular, the ideal $I$ refers here to the augmentation ideal of $k u^{*}\left(B V_{+}\right)$.

Proposition 8.20. The local cohomology of $Q / v$ is concentrated in cohomological degrees 0 and 1 :

$$
\begin{aligned}
& H_{I}^{0}(Q / v) \cong 2 \mathbb{Z} \\
& H_{I}^{1}(Q / v) \cong \mathbb{F}^{\oplus r-1} \oplus D \mathfrak{L}_{1, *} .
\end{aligned}
$$

In particular, $2 H_{I}^{1}(Q / v)=0$.

Proof. The result follows from the exact sequence

$$
0 \rightarrow H_{I}^{0}(Q / v) \rightarrow \mathbb{Z} \rightarrow H_{I}^{1}(\bar{Q} / v) \rightarrow H_{I}^{1}(Q / v) \rightarrow 0 .
$$

The connecting morphism is non-trivial (this can be seen by considering the behaviour modulo 2), whence the result. (This also explains the notation $2 \mathbb{Z}$ ).

The local cohomology of $Q$ can now be analysed by using the exact sequence associated to $Q \stackrel{v}{\rightarrow} Q \rightarrow Q / v$, which has the form

$$
0 \rightarrow H_{I}^{0}(Q) \stackrel{v}{\rightarrow} H_{I}^{0}(Q) \rightarrow H_{I}^{0}(Q / v) \cong 2 \mathbb{Z} \rightarrow H_{I}^{1}(Q) \stackrel{v}{\rightarrow} H_{I}^{1}(Q) \rightarrow H_{I}^{1}(Q / v) \rightarrow 0 .
$$

The calculation of $H_{I}^{0}(Q)$ is straightforward (cf. [BG03, Section 4.4]), and this implies that the image of the connecting morphism is $\mathbb{Z} / 2^{r-1}$. This is sufficient to calculate the local cohomology. A direct approach is taken in [BG03]; the above is preferred here since it stresses the relationship between $H_{I}^{1}(Q / v)$ and the $v$-adic filtration of $H_{I}^{1}(Q)$. 
Proposition 8.21. (cf. [BG03, Lemma 4.5.1]) The associated graded of the $v$-adic filtration of $H_{I}^{1}(Q)$ is given by

$$
v^{i} H_{I}^{1}(Q) / v^{i+1} H_{I}^{1} Q \cong \begin{cases}\left(\mathbb{F}^{\oplus r-i-1} \oplus D \mathfrak{L}_{1, *}\right) \cong H_{I}^{i+2}\left(\mathfrak{L}_{i+2, *}\right) & 0 \leqslant i \leqslant r-3 \\ \mathbb{F} \oplus D \mathfrak{L}_{1, *} & i=r-2 \\ D \mathfrak{L}_{1, *} & i \geqslant r-1 .\end{cases}
$$

Proof. To prove that the naturality with respect to $V$ is correct, use the description $Q\left[\frac{1}{y^{*}}\right] / Q$ that is given in [BG03, Proposition 4.4.7].

\section{Remark 8.22.}

1. Grading shifts are suppressed.

2. [BG03, Lemma 4.5.1] is a statement about the 2-adic filtration; this coincides with the $v$-adic filtration (cf. the final statement of Lemma 3.9).

3. The distinction between the cases $i \leqslant r-3$ and $i=r-2$ is simply to underline the isomorphism with $H_{I}^{i+2}\left(\mathfrak{L}_{i+2, *}\right)$; this is related to the surjection between local cohomology groups given in Corollary 8.18.

\subsection{The local cohomology spectral sequence for $k u_{*}\left(B V_{+}\right)$}

The local cohomology of $k u^{*}\left(B V_{+}\right)$is determined by the short exact sequence

$$
0 \rightarrow \operatorname{tors}_{v} k u^{*}\left(B V_{+}\right) \rightarrow k u^{*}\left(B V_{+}\right) \rightarrow \operatorname{cotors}_{v} k u^{*}\left(B V_{+}\right) \rightarrow 0
$$

and the local cohomology of $\operatorname{tors}_{v} k u^{*}\left(B V_{+}\right)$(cf. Section 8.2) and of $\operatorname{cotors}_{v} k u^{*}\left(B V_{+}\right)$ (cf. Section 8.3).

As in [BG03, Section 4.11], it is illuminating to consider an $E^{1}$-page of the local cohomology spectral sequence that serves to calculate the local cohomology at the $E^{2}$-page.

Theorem 8.23. (cf. [BG03, Section 4.11]) For $V \in \mathrm{Ob} \mathscr{V}^{f}$ of rank $r>1$, there is a local cohomology spectral sequence converging to $k u^{*}\left(B V_{+}\right)$with (up to shift in grading)

$$
E_{s, *}^{1}= \begin{cases}\mathbb{Z}[v] & s=0 \\ H_{I}^{1}(Q) & s=-1 \\ H_{I}^{-s}\left(\mathfrak{L}_{-s, *}\right) & -2 \geqslant s \geqslant 1-r \\ H_{I}^{r}\left(k u^{*}\left(B V_{+}\right)\right) & s=-r\end{cases}
$$

where $H_{I}^{r}\left(k u^{*}\left(B V_{+}\right)\right)$fits into an exact sequence

$$
0 \rightarrow \mathbb{F} \oplus D \mathfrak{L}_{1, *} \rightarrow H_{I}^{r}\left(k u^{*}\left(B V_{+}\right)\right) \rightarrow H_{I}^{r}\left(H \mathbb{Z}^{*}\left(B V_{+}\right)\right)
$$

under the morphism induced by $k u^{*}\left(B V_{+}\right) \rightarrow H \mathbb{Z}^{*}\left(B V_{+}\right)$.

The $E^{\infty}$-page is given by

$$
E_{s, *}^{\infty}= \begin{cases}\mathbb{Z}[v] & s=0 \\ v^{r-1} H_{I}^{1}(Q) & s=-1 \\ 0 & -2 \geqslant s \geqslant 1-r \\ \operatorname{Image}\left\{H_{I}^{r}\left(k u^{*}\left(B V_{+}\right)\right) \rightarrow H_{I}^{r}\left(H \mathbb{Z}^{*}\left(B V_{+}\right)\right)\right\} & s=-r .\end{cases}
$$


Moreover, the morphism $H_{I}^{0}\left(k u^{*}\left(B V_{+}\right)\right) / v \rightarrow H_{I}^{0}\left(H \mathbb{Z}^{*}\left(B V_{+}\right)\right)$is a bijection onto the permanent cycles in the zero column of the local cohomology spectral sequence for $H \mathbb{Z}_{*}\left(B V_{+}\right)$.

Proof. The $E^{1}$-page of the spectral sequence is determined by combining the results of Corollary 8.18, for the contribution from the local cohomology of $\operatorname{tors}_{v} k u^{*}\left(B V_{+}\right)$, and of Section 8.3, for the local cohomology of $Q$. The results of Section 8.2 provide the exact sequence for $H_{I}^{r}\left(k u^{*}\left(B V_{+}\right)\right)$.

The entire behaviour of the spectral sequence is determined by the fact that the only non-trivial differentials originate on the $s=-1$ column, using Proposition 8.21 to relate this to the $v$-adic filtration of $H_{I}^{1}(Q)$.

\section{References}

[Ada74] J.F. Adams, Stable homotopy and generalised homology, Chicago Lectures in Mathematics, University of Chicago Press, Chicago, Ill., 1974, MR 0402720 (53 \#6534).

[BG03] R.R. Bruner and J.P.C. Greenlees, The connective K-theory of finite groups, Mem. Amer. Math. Soc. 165 (2003), no. 785, viii+127, MR 1997161 (2004e:19003).

[BG10] R.R. Bruner and J.P.C. Greenlees, Connective real $K$-theory of finite groups, Mathematical Surveys and Monographs, vol. 169, American Mathematical Society, Providence, RI, 2010, MR 2723113 (2011k:19007).

[BH93] W. Bruns and J. Herzog, Cohen-Macaulay rings, Cambridge Studies in Advanced Mathematics, vol. 39, Cambridge University Press, Cambridge, 1993, MR 1251956 (95h:13020).

[BV00] A. Bak and N. Vavilov, Presenting powers of augmentation ideals and Pfister forms, K-Theory 20 (2000), no. 4, 299-309, Special issues dedicated to Daniel Quillen on the occasion of his sixtieth birthday, Part IV, MR 1803640 (2001k:16047).

[Kuh94a] N.J. Kuhn, Generic representations of the finite general linear groups and the Steenrod algebra. I, Amer. J. Math. 116 (1994), no. 2, 327-360, MR 1269607 (95c:55022).

[Kuh94b] N.J. Kuhn, Generic representations of the finite general linear groups and the Steenrod algebra. II, K-Theory 8 (1994), no. 4, 395-428, MR 1300547 (95k:55038).

[Kuh95] N.J. Kuhn, Generic representations of the finite general linear groups and the Steenrod algebra. III, K-Theory 9 (1995), no. 3, 273-303, MR 1344142 (97c:55026).

[Oss89] E. Ossa, Connective K-theory of elementary abelian groups, Transformation groups (Osaka, 1987), Lecture Notes in Math., vol. 1375, Springer, Berlin, 1989, pp. 269-275, MR 1006699 (90h:55009).

[Pow12] G.M.L. Powell, On connective KO-theory of elementary abelian 2groups, arXiv:1207.6883[math.AT], 2012, to appear in Algebraic and Geometric Topology. 
[PV77] I.B.S. Passi and L.R. Vermani, The associated graded ring of an integral group ring, Math. Proc. Cambridge Philos. Soc. 82 (1977), no. 1, 25-33, MR 0437626 (55 \#10550).

Geoffrey M.L. Powell Geoffrey.Powell@math.cnrs.fr

Laboratoire Angevin de Recherche en Mathématiques, UMR 6093, Faculté des Sciences, Université d'Angers, 2 Boulevard Lavoisier, 49045 Angers, France 